\title{
Sur le caractère gaussien de la convergence presque partout
}

\author{
par \\ Michel Weber (Strasbourg)
}

\begin{abstract}
We establish functional type inequalities linking the regularity properties of sequences of operators $S=\left(S_{n}\right)$ acting on $L^{2}$-spaces with those of the canonical Gaussian process on the associated subsets of $L^{2}$ defined by $\left(S_{n}(f)\right), f \in L^{2}$. These inequalities allow us to easily deduce as corollaries Bourgain's famous entropy criteria in the theory of almost everywhere convergence. They also provide a better understanding of the role of the Gaussian processes in the study of almost everywhere convergence. A partial converse path to Bourgain's entropy criteria is also proposed.
\end{abstract}

1. Introduction, résultats. Soit $(X, \mathcal{A}, \mu)$ un espace probabilisé, et considérons une suite $S$ d'opérateurs continus $S_{n}: L^{2}(\mu) \rightarrow L^{2}(\mu), n=$ $1,2, \ldots$ L'étude des propriétés de convergence presque partout de la suite $\left(S_{n} f\right)_{n \geq 1}$, pour tout $f \in L^{p}(\mu)$, où $2 \leq p<\infty$, est un des thèmes fondamentaux de la théorie ergodique. Ces propriétés font naturellement intervenir les opérateurs de maximum associés à la suite $S$ :

$$
\forall I \subset \mathbb{N}, \quad S_{I}(g)=\sup _{n \in I}\left|S_{n}(g)\right|, \quad S^{*}(g)=\sup _{n \geq 1}\left|S_{n}(g)\right| .
$$

D'après les principes généraux de la théorie ergodique que sont le principe de Banach et le principe de continuité ([K], théorèmes 7.2 , p. 64 et 7.8 , p. 68), l'ensemble des éléments $f \in L^{2}(\mu)$ pour lesquels la suite $\left(S_{n}(f)\right)_{n \geq 1}$ converge $\mu$-presque partout est fermé dans $L^{2}(\mu)$ si, et seulement si, il existe une fonction $C: \mathbb{R}^{+} \rightarrow \mathbb{R}^{+}$décroissante telle que $\lim _{\alpha \rightarrow \infty} C(\alpha)=0$ et pour laquelle $\mu\left\{S^{*}(f)>\alpha\|f\|_{2}\right\} \leq C(\alpha)\left(\alpha>0, f \in L^{2}(\mu)\right)$. Lorsqu'en outre, la suite $S$ commute avec une famille $\mathcal{E}$ de transformations mesurables de $X$ préservant $\mu$, et mélangeante au sens suivant :

2000 Mathematics Subject Classification: Primary 28D05, 60G15; Secondary 26D07, $60 \mathrm{G} 17$.

Key words and phrases: almost sure convergence, Gaussian processes, entropy numbers. 


$$
\forall A, B \in \mathcal{A}, \forall \alpha>1, \exists T \in \mathcal{E}: \quad \mu\left(A \cap T^{-1} B\right) \leq \alpha \mu(A) \mu(B),
$$

alors en vertu du second principe $C(\alpha)=O\left(\alpha^{-2}\right)$. C'est en particulier vrai si $S$ commute avec un endomorphisme ergodique de $(X, \mathcal{A}, \mu)$. De sorte que l'étude de la convergence presque partout de la suite $S$ pour tout $f \in L^{2}$ se ramène, modulo des hypothèses de commutation adéquates, à établir l'existence d'une inégalité maximale $\mu\left\{S^{*}(f)>\alpha\|f\|_{2}\right\} \leq C \alpha^{-2}$, ainsi que celle d'une classe dense dans $L^{2}(\mu)$ pour laquelle il y a convergence presque partout.

Dans ce qui suit, on notera $Z=\left\{Z_{h}: h \in H\right\}$ le processus gaussien canonique sur $L^{2}(\mu)$, c'est-à-dire, le processus gaussien $Z=\left\{Z_{h}: h \in H\right\}$ ayant pour fonction de covariance $\Gamma\left(h, h^{\prime}\right)=\left\langle h, h^{\prime}\right\rangle, h, h^{\prime} \in H$. Dans [Bo1] (voir aussi [W2]), Bourgain a établi un lien remarquable entre les propriétés de convergence presque partout de la famille $S=\left(S_{n}\right)_{n}$ sur l'espace $L^{p}(\mu)$ $(2 \leq p \leq \infty)$ et la régularité de $Z$ sur les ensembles $C_{g}=\left(S_{n}(g)\right) \subset L^{2}(\mu)$. Ce lien se traduit par le fait que ces ensembles sont des $G B$-ensembles, i.e. $\mathbb{E} \sup _{n>1}\left|Z\left(S_{n}(f)\right)\right|<\infty$, et fournit donc, via la minoration de Sudakov (inégalité $(2.7)$ ), des conditions nécessaires sur l'entropie métrique des ensembles $C_{g}$ pour que les propriétés de convergence presque partout de $S$ (relativement à $L^{p}(\mu), 2 \leq p \leq \infty$, voir corollaires 1.2 et 1.7) aient lieu. Ces conditions, signifiant que ces ensembles sont tous pas trop épais, se sont révélées être un outil remarquablement efficace et fondamental pour établir des résultats de type négatif dans l'étude de problèmes classiques de convergence presque partout ([Bo1]).

Dans cet article, nous proposons tout d'abord de développer cette approche en montrant que les critères d'entropie de Bourgain se déduisent simplement d'inégalités fonctionnelles liant la famille $S$ et le processus gaussien canonique $Z$. Introduisons à cet effet les fonctionnelles maximales suivantes de la suite $S$ sur $I$ :

$$
\boldsymbol{\Omega}_{1}(S, I)=\sup _{\|g\|_{2, \mu} \leq 1}\left\|S_{I}(g)\right\|_{1, \mu}, \quad \boldsymbol{\Omega}_{2}(S, I)=\sup _{\|g\|_{2, \mu} \leq 1}\left\|S_{I}(g)\right\|_{2, \mu} .
$$

De même, on note $\boldsymbol{\Omega}_{i}^{*}(S)=\boldsymbol{\Omega}_{i}(S, \mathbb{N}), i=1,2$. Lorsque la suite $S$ est une suite d'opérateurs $L^{2}(\mu)-L^{\infty}(\mu)$ continus, il sera utile de considérer aussi les fonctionnelles suivantes introduites par Bourgain dans [Bo1] et Bellow-Jones dans $[\mathrm{BJ}]$ :

$$
\begin{aligned}
\boldsymbol{\Omega}_{\infty, 2}(S, I, \varepsilon) & =\sup _{\|g\|_{\infty, \mu} \leq 1,\|g\|_{2, \mu} \leq \varepsilon} \int S_{I}(g) d \mu, \\
\boldsymbol{\Omega}_{\infty, 2}^{*}(S, \varepsilon) & =\sup _{\|g\|_{\infty, \mu} \leq 1,\|g\|_{2, \mu} \leq \varepsilon} \int S^{*}(g) d \mu .
\end{aligned}
$$

Lorsque de plus les opérateurs $S_{n}$ sont continus sur $L^{p}(\mu)$, pour un réel $p \in[2, \infty]$, nous posons pour tout ensemble fini $I$ d'entiers, et $g \in L^{p}(\mu)$, 


$$
S(I, p)=\sup _{n \in I}\left\|S_{n}\right\|_{p}, \quad\left\|S_{n}\right\|_{p}=\sup _{\|g\|_{p, \mu} \leq 1}\left\|S_{n}(g)\right\|_{p, \mu}
$$

Posons aussi

$$
\begin{gathered}
\boldsymbol{\Phi}(S, I)=\sup _{\|f\|_{2, \mu} \leq 1} \mathbb{E} \sup _{n \in I} Z\left(S_{n}(f)\right), \\
\boldsymbol{\Phi}_{a}(S, I)=\sup _{\|f\|_{2, \mu} \leq 1} \mathbb{E} \sup _{n \in I}\left|Z\left(S_{n}(f)\right)\right| .
\end{gathered}
$$

Nous établissons des inégalités de liaison directe entre les fonctionnelles $\boldsymbol{\Omega}_{\infty, 2}(S, I, \varepsilon), \boldsymbol{\Omega}_{2}(S, I)$ et $\boldsymbol{\Phi}_{a}(S, I)$. Le théorème ci-dessous illustre bien notre propos. Soit $S$ une suite de $L^{2}(\mu)$ contractions telle que

$$
c=\inf _{n \geq 1}\left\|S_{n}\right\|>0 \text {. }
$$

Supposons

(H1) il existe une suite $\left\{T_{j}: j \geq 1\right\}$ d'isométries positives de $L^{2}(\mu)$, préservant 1 , commutant avec la suite $\left\{S_{n}: n \geq 1\right\}$ et vérifiant sur $L^{\infty}(\mu)$ le théorème ergodique dans $L^{1}(\mu)$ :

$$
\forall f \in L^{\infty}(\mu), \quad \lim _{J \rightarrow \infty}\left\|J^{-1} \sum_{j \leq J} T_{j} f-\int f d \mu\right\|_{1, \mu}=0 .
$$

Supposons aussi que $S$ vérifie une inégalité maximale sur $L^{2}(\mu)$ :

$$
\forall f \in L^{2}(\mu), \quad\left\|S^{*}(f)\right\|_{2, \mu} \leq C\|f\|_{2, \mu} .
$$

Alors

ThÉORÈme 1.1. Pour toute partie $I$ de $\mathbb{N}$,

$$
C_{1} \boldsymbol{\Phi}_{a}(S, I) \leq \boldsymbol{\Omega}_{2}(S, I) \leq C_{2} \frac{\boldsymbol{\Phi}_{a}(S, I)}{S(I, 2)} \leq C_{2} \frac{\boldsymbol{\Phi}_{a}(S, I)}{c}
$$

avec $C_{1}=1 / 2, C_{2}=\sqrt{\pi / 2} C$.

Remarque. - L'inégalité de gauche est vraie sans l'hypothèse (HM). Les constantes $c, C$ sont calculables dans de nombreux exemples classiques : moyennes ergodiques associées aux rotations du tore, sommes de Riemann, sommes partielles trigonométriques etc.

- Pour $\boldsymbol{\Omega}_{1}(S, I)$, on dispose d'un encadrement semblable :

$$
C_{1} \boldsymbol{\Phi}(S, I) \leq \boldsymbol{\Omega}_{1}(S, I) \leq C_{2} \boldsymbol{\Phi}_{a}(S, I),
$$

avec $C_{1}=1, C_{2}=\sqrt{\pi / 2} C$.

Convenons de noter pour tout $h \in L^{1}(\mu), \mu(h)=\langle h, 1\rangle$. Dans le corollaire qui suit, nous remplacerons l'hypothèse (H1) par une hypothèse légèrement plus fine : 
(H2) il existe une famille $\mathcal{E}=\left\{T_{j}: j \geq 1\right\}$ de transformations ponctuelles de $X$, préservant $\mu$, commutant avec les $S_{n}\left(S_{n} T_{j}(f)=T_{j} S_{n}(f)\right)$ et ergodique :

$$
\forall f, g \in L^{2}(\mu), \quad \lim _{n \rightarrow \infty} \frac{1}{n} \sum_{k=1}^{n}\left\langle T_{k} f, g\right\rangle=\mu(f) \mu(g) .
$$

Sous cette hypothèse, la propriété $(\mathcal{E})$ est vérifiée. La suite $S$ vérifie par conséquent les conditions d'application du principe de continuité.

Corollaire 1.2 (Critère d'entropie métrique dans $L^{p}(\mu)$, [Bo1], Proposition 1). Soit $S$ une suite d'opérateurs continus $S_{n}: L^{2}(\mu) \rightarrow L^{2}(\mu), n=$ $1,2, \ldots$, vérifiant l'hypothèse $(\mathrm{H} 2)$, et tels que pour un réel $p \in[2, \infty)$, la condition suivante soit réalisée :

$$
\forall f \in L^{p}(\mu), \quad \sup _{n \geq 1}\left|S_{n}(f)\right|<\infty, \quad \mu \text {-presque partout. }
$$

Alors, pour tout $f \in L^{p}(\mu)$, les ensembles $C_{f}$ sont des $G B$-ensembles de $L^{2}(\mu)$.

En particulier, il existe une constante numérique $C_{1}$ et une constante $C_{2}$ dépendant de la suite $S$ telles que pour tout $f \in L^{p}(\mu)$,

$$
C_{1} \sup \left\{\varepsilon \sqrt{\log N_{f}(\varepsilon)}, \varepsilon>0\right\} \leq \mathbb{E} \sup _{n \geq 1} Z\left(S_{n}(f)\right) \leq C_{2}\|f\|_{2},
$$

où pour tout $\varepsilon>0$, nous notons par $N_{f}(\varepsilon)$ le cardinal minimal d'un recouvrement de $C_{f}$ par des $L^{2}(\mu)$-boules ouvertes, de rayon $\varepsilon$ et centrées dans $C_{f}$.

La première inégalité en (1.5) fournit une estimation entropique optimale lorsque la suite $S_{n}$ est une suite de produits de convolutions (cf. [W4], remarque 4.1.4); la seconde inégalité indique que les ensembles $C_{f}$ sont uniformément $G B$.

Démonstration. Comme l'hypothèse (H2) implique (H1), en vertu du principe de continuité, on sait que

$$
\sup _{\|f\|_{2, \mu} \leq 1} \sup _{t \geq 0} t^{2} \mu\left\{S^{*}(f)>t\|f\|_{2, \mu}^{2}\right\}<\infty .
$$

Donc, $\boldsymbol{\Omega}_{1}^{*}(S)<\infty$, d'où $\boldsymbol{\Phi}(S, \mathbb{N})<\infty$.

Le théorème 1.1 permet aussi de quantifier $\boldsymbol{\Omega}_{1}(S, I), \boldsymbol{\Omega}_{2}(S, I)$, ce qui n'est pas possible à l'aide du critère d'entropie précédent. Voici une application à l'étude des sommes de Riemann. Définissons pour tout entier $n=1,2, \ldots$ l'opérateur somme de Riemann d'ordre $n$ de $f$ comme suit :

$$
\forall x \in \Pi=\mathbb{R} / \mathbb{Z}, \quad R_{n}(f)(x)=\frac{1}{n} \sum_{0 \leq j<n} f\left(x+\frac{j}{n}\right) .
$$


Notons $m$ la mesure de Lebesgue sur $\Pi$. Considérons maintenant la suite $P_{1}, P_{2}, \ldots$ des nombres premiers, et posons aussi

$$
\forall x \in \Pi, \forall s \geq 1, \quad A_{s}(f)(x)=\frac{1}{s} \sum_{i=1}^{s} R_{P_{i}}(f)(x) .
$$

Proposition 1.3. Il existe une constante absolue c telle que

$$
\begin{aligned}
& \forall r \geq 1, \quad \sup _{\|f\|_{2, m} \leq 1} \int_{i=1} \sup _{i=1}^{r}\left|R_{P_{i}}(f)\right| d m \geq c \sqrt{\log r}, \\
& \forall s \geq 1, \quad \sup _{\|f\|_{2, m} \leq 1} \int \sup _{i=1}^{s}\left|A_{2^{i}}(f)\right| d m \geq c \sqrt{\log s .}
\end{aligned}
$$

Démonstration. Considérons l'ensemble d'entiers

$$
E=\left\{P_{1}^{\alpha_{1}} \ldots P_{r}^{\alpha_{r}}: \alpha_{i} \in\{0,1\}\right\}
$$

et la fonction

$$
f(x)=2^{-r / 2} \sum_{n \in E} e^{2 i \pi n x} .
$$

Alors

$$
R_{P_{s}}(f)(x)=2^{-r / 2} \sum_{n \in E \cap P_{s} \mathbb{Z}} e^{2 i \pi n x},
$$

et pour $1 \leq s \neq t \leq r$,

$$
\left\|R_{P_{s}}(f)-R_{P_{t}}(f)\right\|_{2}=1 / \sqrt{2} .
$$

Il résulte donc du théorème 1.1 et de la minoration de Sudakov (inégalité (2.7)) que

$$
\sup _{\|f\|_{2, m} \leq 1} \int_{i=1} \sup _{i=1}^{r}\left|R_{P_{i}}(f)\right| d m \geq \frac{B}{\sqrt{2}} \sqrt{\log r},
$$

où $B$ est une constante numérique, d'où le résultat.

Montrons à présent l'inégalité relative aux moyennes des sommes de Riemann. Soit $u$ fixé et $2 \leq s<t \leq u+1$, et introduisons les éléments suivants :

$$
\begin{gathered}
E=E_{u}=\left\{N_{1}^{\alpha_{1}} \ldots N_{u}^{\alpha_{u}}: \alpha_{i} \in\{0,1\}\right\}, \quad N_{j}=P_{2^{j}+1} \ldots P_{2^{j+1}} \text { pour } j \geq 1, \\
f(x)=f_{u}(x)=\frac{1}{\left|E_{u}\right|^{1 / 2}} \sum_{n \in E_{u}} e^{2 i \pi n x} .
\end{gathered}
$$

Il résulte de la démonstration du théorème 1.3 de $[\mathrm{RW}]$ que

$$
\inf _{2 \leq s<t \leq u+1}\left\|A_{2^{s}} f-A_{2^{t}} f\right\|_{2} \geq \frac{1}{2 \sqrt{3}} .
$$

Le théorème 1.1 et la minoration de Sudakov permettent de conclure. 
Définissons à présent pour toute fonction $f: \Pi \rightarrow \mathbb{R}$ les opérateurs suivants :

$$
\forall n \geq 1, \quad S_{n} f(x)=\frac{1}{n} \sum_{j \leq n} f(j x) .
$$

Proposition 1.4. Pour tout entier $M \geq 26$,

$$
\sup _{\|f\|_{2} \leq 1} \int_{1 \leq i \leq M} \sup _{1 \leq S^{i}} f \mid d \mu \geq \frac{B}{8} \sqrt{\log (M / 26)},
$$

où la constante $B$ est celle de l'inégalité (2.7).

Démonstration. Soit $s \in \mathbb{N}$ fixé. Soit $P_{1}, P_{2}, \ldots$ la suite des nombres premiers; on pose, pour tout $T \geq 0$,

$$
A_{T}=\left\{n=P_{1}^{\alpha_{1}} \ldots P_{s}^{\alpha_{s}}: 2^{T} \leq n<2^{T+1}, \alpha_{i} \geq 0, i=1, \ldots, s\right\} .
$$

Choisissons $d$ tel que $2^{d} \leq P_{s}$, puis $T=[[d / 2] / 13]$. Posons

$$
f_{s}(x)=\frac{1}{\sharp\left(A_{T}\right)^{1 / 2}} \sum_{n \in A_{T}} e^{2 i \pi n x} .
$$

La démonstration de Bourgain dans [Bo1] (voir aussi [W4], Chapitre V, par. 5.1.2 pour des calculs détaillés) montre que

$$
N\left(\left(S_{4^{i}}\left(f_{s}\right), i \leq[d / 2]\right), 1 / 4\right) \geq[[d / 2] / 13] .
$$

Il résulte donc du théorème 1.1 et de l'inégalité (2.7) que pour tout $M \geq 26$,

$$
\sup _{\|f\|_{2} \leq 1} \int_{1 \leq i \leq M} \sup _{1 \leq S_{4^{i}}} f \mid d \mu \geq \frac{B}{8} \sqrt{\log (M / 26)} .
$$

Nous établirons aussi le résultat suivant relatif à la fonctionnelle maximale de Bellow-Jones.

ThÉORÈme 1.5. Supposons que pour tout $n \geq 1, S_{n}$ soit $L^{2}(\mu)-L^{\infty}(\mu)$ continu, et qu'en outre l'hypothèse (H1) soit satisfaite. Alors, pour toute partie finie $I$ de $\mathbb{N}$, tout $A>0$ et tout $R>0$,

$$
\boldsymbol{\Phi}(S, I) \leq \sqrt{2 \sharp(I)} S(I, 2) e^{-A^{2} / 8}+\sqrt{2} A S(I, \infty) e^{-R^{2} / 4}+A \boldsymbol{\Omega}_{\infty, 2}(S, I, R / A) .
$$

Ce théorème a pour conséquence

Proposition 1.6. Soit $\left\{S_{n}: n \geq 1\right\}$ une suite de $L^{2}(\mu)-L^{\infty}(\mu)$ contractions vérifiant l'hypothèse (H1). Posons pour tout entier $K>0$,

$$
\Phi^{*}(S, K)=\sup _{\substack{\|f\|_{2, \mu} \leq 1 \\ I \subset \mathbb{N}, \sharp(I)=K}} \mathbb{E} \sup _{n \in I} Z\left(S_{n}(f)\right) .
$$

Alors, pour tout réel $\varrho>0$, il existe un réel $0<C_{\varrho}<\infty$ tel que pour tout entier $K \geq 3$ et tout $R>0$,

$$
\frac{\boldsymbol{\Phi}^{*}(S, K)}{\sqrt{\log K}} \leq C_{\varrho}\left(K^{-\varrho}+e^{-R^{2} / 4}\right)+\boldsymbol{\Omega}_{\infty, 2}^{*}(S, R / \sqrt{\log K}) .
$$


En particulier,

$$
\limsup _{K \rightarrow \infty} \frac{\boldsymbol{\Phi}^{*}(S, K)}{\sqrt{\log K}} \leq \lim _{\varepsilon \rightarrow 0} \boldsymbol{\Omega}_{\infty, 2}^{*}(S, \varepsilon) .
$$

Démonstration. Le théorème 1.5 implique

$$
\boldsymbol{\Phi}(S, I) \leq \sqrt{2 \sharp(I)} e^{-A^{2} / 8}+\sqrt{2} A e^{-R^{2} / 4}+A \boldsymbol{\Omega}_{\infty, 2}(S, I, R / A) .
$$

Soit $\varrho>0$ fixé; choisissons $C=8 \varrho+4$. Soit $K \geq 3$. Posons $A=\sqrt{C \log K}$. Alors, pour toute partie $I$ de $\mathbb{N}$ telle que $\sharp(I)=K$,

$$
\frac{\boldsymbol{\Phi}(S, I)}{\sqrt{\log K}} \leq \frac{\sqrt{2}}{\sqrt{\log K}} K^{-\varrho}+\sqrt{2 C} e^{-R^{2} / 4}+\boldsymbol{\Omega}_{\infty, 2}^{*}\left(S, \frac{R}{\sqrt{\log K}}\right),
$$

puisque $\sqrt{C} \boldsymbol{\Omega}_{\infty, 2}^{*}(S, R / \sqrt{C \log K}) \leq \boldsymbol{\Omega}_{\infty, 2}^{*}(S, R / \sqrt{\log K})$ (l'inégalité inverse est aussi vraie pour $C \geq 1$ ). D'où (1.7) avec $C_{\varrho}=\sqrt{2 C}$, en prenant le maximum sur toutes les parties $I$ de $\mathbb{N}$ telles que $\sharp(I)=K$.

On obtient sans peine l'inégalité (1.8) à partir de (1.7) en laissant $R$ décrire une suite croissante d'entiers $\left(R_{K}\right)$ telle que $\lim _{K \rightarrow \infty} R_{K}=\infty$ et $\lim _{K \rightarrow \infty} R_{K} / \sqrt{\log K}=0$, puis en faisant tendre $\varrho$ vers zéro.

Nous en déduisons par exemple comme corollaire

Corollaire 1.7 (Critère d'entropie dans $L^{\infty}$, [Bo1], Proposition 2). Soit $\left\{S_{n}: n \geq 1\right\}$ une suite de $L^{2}(\mu)-L^{\infty}(\mu)$ contractions vérifiant l'hypothèse (H1). Supposons aussi que

$$
\forall f \in L^{\infty}(\mu), \quad \mu\left\{\left\{S_{n}(f): n \geq 1\right\} \text { converge }\right\}=1 .
$$

Alors,

$$
\forall \delta>0, \quad C(\delta)=\sup _{f \in L^{\infty}(\mu),\|f\|_{2}=1} N_{f}(\delta)<\infty .
$$

Démonstration. Supposons qu'il existe un réel $\delta>0$ tel que $C(\delta)=\infty$. Alors, pour tout entier $K \geq 3$, il existe $f \in L^{\infty}(\mu)$ telle que $\|f\|_{2, \mu}=1$ et $I$ avec $\sharp(I)=K$ tels que

$$
\inf _{\substack{n, m \in I \\ n \neq m}}\left\|S_{n}(f)-S_{m}(f)\right\|_{2, \mu} \geq \delta .
$$

Il en résulte, en vertu de l'inégalité (2.7) d'une part, et de la proposition 1.6 (avec $\varrho=1$ et $C=C_{\varrho}$ ) d'autre part, que

$$
B \delta \leq C\left(K^{-1}+e^{-R^{2} / 4}\right)+\Omega_{\infty, 2}^{*}(S, R / \sqrt{\log K}),
$$

où $B$ est une constante numérique. En choisissant alors $R$ tel que $C e^{-R^{2} / 4} \leq$ $\frac{1}{2} B \delta$, puis en faisant tendre $K$ vers l'infini, on en déduit

$$
\frac{1}{2} B \delta \leq \limsup _{\varepsilon \rightarrow 0} \Omega_{\infty, 2}^{*}(S, \varepsilon) \text {. }
$$


Ceci apporte une contradiction, puisqu'en vertu de la proposition 2.8, on sait que l'opérateur maximal $\boldsymbol{\Omega}_{\infty, 2}^{*}(S, \varepsilon)$ est continu en 0 . D'où le corollaire.

Les critères d'entropie de Bourgain ont montré que les propriétés de bornitude presque sûre, ou de convergence presque sûre de la suite $S$, étaient "contrôlées" par celles du processus gaussien canonique $Z$ sur des ensembles intrinsèquement liés à $S$. Les théorèmes 1.1 et 1.5 fournissent quant à eux des quantifications gaussiennes des opérateurs maximaux liés à $S$, desquelles découlent ces critères. Il est donc tentant et naturel à la fois de chercher à comprendre jusqu'où peut s'exercer ce phénomème de "couplage de régularité". Ceci est d'autant plus naturel que dans bien des situations les ensembles $C_{f}=\left\{S_{n}(f): n \geq 1\right\}$ ont une structure gaussienne très simple (ce sont souvent des $G B$-ensembles). Le cas des sommes partielles d'une série trigonométrique est à cet égard très symptomatique, puisque les ensembles $C_{f}$ sont de la forme $\left\{a_{k}:|k| \leq n\right\}_{n}$ avec $a=\left(a_{k}\right)_{k} \in \ell_{2}$, ce qui en fait trivialement des $G B$-ensembles. Dans cette optique, la question suivante est naturelle :

Question. Soit $f \in L^{2}(\mu)$ telle que $\mathbb{E} \sup _{n>1}\left|Z\left(S_{n}(f)\right)\right|<\infty$. Que peut-on en inférer sur la régularité de la suite $\left(S_{n}(f)\right)_{n}$ ?

Cette question a été étudiée dans des travaux antérieurs [W1]-[W3] à l'aide des propriétés de compacité relative des lois des éléments de Stein $\left(F_{J, f}\right)_{J}$ définis en $(2.8)$ et naturellement associés à tout $f \in L^{2}$. Cet outil est particulièrement bien adapté (mais aussi quasi-exclusivement) au cas des rotations (i.e. la suite d'isométries $\left(T_{j}\right)$ est la suite des puissances de l'opérateur unitaire associé à une rotation irrationnelle du tore $\Pi$. Dans ce cas, quel que soit $f \in L^{2}(\Pi)$, la suite des lois de $\left(F_{J, f}\right)_{J}$ est relativement compacte dans $L^{2}(\Pi)$. On peut alors avantageusement remplacer la suite $\left(F_{J, f}\right)_{J}$ par une de ses limites en loi, aisément calculable à partir du développement en série de Fourier de $f$. Si donc $\mathbb{E} \sup _{n \geq 1}\left|Z\left(S_{n}(f)\right)\right|<\infty$, et si en outre les coefficients de Fourier de $f$ sont tous non nuls, on en déduit l'existence d'inégalités maximales sur l'espace de Hilbert autoreproduisant associé ([W3], Théorème 4.4, p. 596) et aussi le fait que l'ensemble $\mathcal{G}=\left\{g \in L^{2}(\Pi): \mu\left\{S^{*}(g)<\infty\right\}=1\right\}$ est partout dense dans $L^{2}(\Pi)$. Un résultat analogue a lieu pour la convergence presque sûre.

Evidemment la question-clé se trouve concentrée dans l'existence ou non d'une inégalité maximale lorsque $\mathbb{E} \sup _{n \geq 1}\left|Z\left(S_{n}(f)\right)\right|<\infty$ pour tout $f \in$ $L^{2}(\mu)$. Nous n'avons pas de réponse définitive à apporter à ce problème. Par contre, nous démontrerons le résultat partiel ci-dessous, significatif à cause de la quantité $\zeta(\Delta, f)$ qui y figure.

ThÉORÈme 1.8. Soit $S$ une suite d'opérateurs continus $S_{n}: L^{2}(\mu) \rightarrow$ $L^{2}(\mu), n=1,2, \ldots$, vérifiant l'hypothèse suivante: 
$\left(\mathrm{H} 2^{\prime}\right) \quad$ il existe une famille $\mathcal{E}=\left\{T_{j}: j \geq 1\right\}$ de transformations ponctuelles de $X$, préservant $\mu$, commutant avec les $S_{n}\left(S_{n} T_{j}(f)=T_{j} S_{n}(f)\right)$ et faiblement mélangeante :

( $\forall) \quad \forall f, g \in L^{2}(\mu), \quad \lim _{n \rightarrow \infty} \frac{1}{n} \sum_{k=1}^{n}\left|\left\langle T_{k} f, g\right\rangle-\mu(f) \mu(g)\right|=0$.

Soit $f \in L^{2}(\mu)$. Soit $\Delta$ une partie finie de $\mathbb{N}$. Notons

$$
\zeta(\Delta, f)=\mathbb{E} \sup _{n \in \Delta}\left|Z\left(S_{n}(f)\right)\right| .
$$

Alors il existe une constante absolue $K$ et un nombre fini $t(\Delta, f)$ tels que

$$
\forall t \geq t(\Delta, f), \quad \mu\left\{S_{\Delta}(f)>t\right\} \leq K \zeta(\Delta, f)^{2} / t^{2} .
$$

On vérifie facilement à l'aide de l'inégalité de Jensen que

$$
\zeta(\Delta, f) \leq \sqrt{\log \sharp(\Delta)} \sup _{n \in \Delta}\left\|S_{n}(f)\right\|_{2, \mu} .
$$

Ceci montre finesse de la majoration précédente pour $t$ grand.

\section{Lemmes préliminaires}

2.1. Commençons par un premier lemme utile, que nous démontrons en détail, pour la commodité du lecteur.

Lemme 2.1. Soit $T$ une isométrie positive de $L^{2}(\mu)$ telle que $T 1=1$.

(a) Alors pour tout $f \in L^{\infty}(\mu)$,

$$
\|T f\|_{\infty, \mu} \leq\|f\|_{\infty, \mu} \quad \text { et } \quad \mu\left\{(T f)^{2}=T f^{2}\right\}=1 .
$$

(b) Si en outre, $T$ est un opérateur continu sur $L^{1}(\mu)$, alors pour tout $f \in L^{2}(\mu)$,

$$
\mu\left\{(T f)^{2}=T f^{2}\right\}=1,
$$

et $T$ est une isométrie positive de $L_{+}^{1}(\mu)$.

(c) Réciproquement, si $T$ est une isométrie positive de $L_{+}^{1}(\mu)$ telle que pour tout $f \in L^{2}(\mu), \mu\left\{(T f)^{2}=T f^{2}\right\}=1$, alors $T 1=1$ et $T$ est une isométrie positive de $L^{2}(\mu)$.

Démonstration. La première assertion est immédiate puisque $T f \leq T 1$. $\|f\|_{\infty, \mu}=\|f\|_{\infty, \mu}$. Soit $A \in \mathcal{F}, 0<\mu(A)<1$. Nous utiliserons la propriété suivante : deux éléments $f, g \in L^{2}(\mu)$ avec $f \geq 0, g \geq 0$ sont à supports disjoints si et seulement si

$$
\|f+g\|_{2, \mu}^{2}=\|f\|_{2, \mu}^{2}+\|g\|_{2, \mu}^{2} .
$$

Cette propriété reste vraie $\left([\mathrm{K}]\right.$, p. 186) dans $L^{p}(\mu)$ avec $1<p<\infty$. Puisque $T$ est une isométrie positive, de $T 1=1$ on déduit que $T \mathbf{1}_{A}$ et $T \mathbf{1}_{A^{c}}$ sont donc à supports disjoints, et $0 \leq T \mathbf{1}_{A}, T \mathbf{1}_{A^{c}} \leq 1$. Soit $E=\left\{0<T \mathbf{1}_{A}<1\right\}=\{0<$ $\left.T \mathbf{1}_{A^{\mathrm{c}}}<1\right\}$. Comme $E \subset \operatorname{supp}\left(T \mathbf{1}_{A}\right) \cap \operatorname{supp}\left(T \mathbf{1}_{A^{\mathrm{c}}}\right)$, on conclut que $T \mathbf{1}_{A}$ et 
$T \mathbf{1}_{A^{c}}$ sont des indicatrices. Par suite, toute fonction simple est transformée par $T$ en une fonction simple. Pour ces fonctions on a $(T f)^{2}=T f^{2}$. Soit $f \in L^{\infty}(\mu), f \geq 0$. Posons pour tout entier $n>\|f\|_{\infty, \mu}$,

$$
\begin{aligned}
f_{n} & =\sum_{q=1}^{n 2^{n}} \frac{q}{2^{n}} \mathbf{1}_{\left\{(q-1) / 2^{n} \leq f<q / 2^{n}\right\}}, \\
g_{n} & =\sum_{q=1}^{n 2^{n}} \frac{q-1}{2^{n}} \mathbf{1}_{\left\{(q-1) / 2^{n} \leq f<q / 2^{n}\right\}} .
\end{aligned}
$$

Alors $f \leq f_{n} \leq f+1 / 2^{n}$ et $g_{n} \leq f \leq g_{n}+1 / 2^{n}$ en tout point. D'une part, par positivité de $T$, et puisque $T 1=1$,

$$
(T f)^{2} \leq\left(T f_{n}\right)^{2}=T f_{n}^{2} \leq T\left(f+\frac{1}{2^{n}}\right)^{2}=T f^{2}+2^{-n+1} T f+2^{-2 n} .
$$

Par suite, $(T f)^{2} \leq T f^{2}$.

D'autre part,

$$
\begin{aligned}
T f^{2} & \leq T\left(g_{n}+\frac{1}{2^{n}}\right)^{2}=T g_{n}^{2}+2^{-n+1} T g_{n}+2^{-2 n} \\
& \leq(T f)^{2}+2^{-n+1} T f+2^{-2 n}
\end{aligned}
$$

D'où aussi, $T f^{2} \leq(T f)^{2}$; et donc $T f^{2}=(T f)^{2}$. Soit maintenant $f \in$ $L^{\infty}(\mu), f=f^{+}-f^{-}$. Comme

$$
\begin{aligned}
\left\|T f^{+}-T f^{-}\right\|_{2, \mu}^{2} & =\|T f\|_{2, \mu}^{2}=\|f\|_{2, \mu}^{2}=\left\|f^{+}\right\|_{2, \mu}^{2}+\left\|f^{-}\right\|_{2, \mu}^{2} \\
& =\left\|T f^{+}\right\|_{2, \mu}^{2}+\left\|T f^{-}\right\|_{2, \mu}^{2}
\end{aligned}
$$

il en ressort que $T f^{+}$et $T f^{-}$sont à supports disjoints. Ceci implique alors

$$
(T f)^{2}=\left(T f^{+}\right)^{2}+\left(T f^{-}\right)^{2}=T\left(f^{+}\right)^{2}+T\left(f^{-}\right)^{2}=T f^{2} .
$$

On a donc établi l'assertion (a) du lemme.

Montrons (b). Soit $f \in L^{2}(\mu)$; il existe une suite $\left(f_{n}\right) \subset L^{\infty}(\mu)$ telle que $\left\|f-f_{n}\right\|_{2} \rightarrow 0$ quand $n \rightarrow \infty$. En vertu de l'inégalité de Cauchy-Schwarz, on a aussi $\left\|f^{2}-f_{n}^{2}\right\|_{1} \rightarrow 0$ quand $n \rightarrow \infty$. Alors

$$
\begin{aligned}
\left\|(T f)^{2}-T f^{2}\right\|_{1} & \leq\left\|(T f)^{2}-\left(T f_{n}\right)^{2}\right\|_{1}+\left\|\left(T f_{n}\right)^{2}-T f_{n}^{2}\right\|_{1}+\left\|T\left(f_{n}^{2}-f^{2}\right)\right\|_{1} \\
& \leq\left\|T\left(f_{n}-f\right)\right\|_{2} \cdot\left\|T\left(f_{n}+f\right)\right\|_{2}+\left\|T\left(f_{n}^{2}-f^{2}\right)\right\|_{1} \rightarrow 0
\end{aligned}
$$

quand $n \rightarrow \infty$ puisque $T$ est continu sur $L^{1}(\mu)$ et $L^{2}(\mu)$. D'où (b).

Enfin (c) est immédiate.

2.2. Rappelons à présent l'outil fondamental de la théorie des processus gaussiens que constitue le lemme de comparaison de Slépian. 
Lemme 2.2 ([LT], théorème 3.15, p. 78). Soit T un ensemble fini; soient $X=\left\{X_{t}: t \in T\right\}$ et $Y=\left\{Y_{t}: t \in T\right\}$ deux processus gaussiens. Supposons que

$$
\forall s, t \in T \times T, \quad\left\|X_{s}-X_{t}\right\|_{2} \leq\left\|Y_{s}-Y_{t}\right\|_{2} .
$$

Alors, pour toute fonction convexe positive croissante $f$ sur $\mathbb{R}^{+}$,

$$
\mathbb{E} f\left[\sup _{T \times T}\left(X_{s}-X_{t}\right)\right] \leq \mathbb{E} f\left[\sup _{T \times T}\left(Y_{s}-Y_{t}\right)\right] .
$$

En particulier,

$$
\mathbb{E} \sup _{t \in T} X_{t} \leq \mathbb{E} \sup _{t \in T} Y_{t} .
$$

Une des conséquences importantes de ce lemme est la minoration suivante de Sudakov : il existe une constante universelle $B$ telle que pour tout processus gaussien $X=\left\{X_{t}: t \in T\right\}$ d'espace d'épreuve $(\Omega, \mathcal{B}, \mathbb{P})$,

$$
\mathbb{E} \sup _{t \in T} X_{t} \geq B \inf _{\substack{s, t \in T \\ s \neq t}}\left\|X_{s}-X_{t}\right\|_{2, \mathbb{P}} \sqrt{\log \operatorname{Card}(T)} .
$$

Soit à présent $g_{1}, g_{2}, \ldots$ une suite isonormale définie sur un espace d'épreuves différent de $(X, \mathcal{A}, \mu)$, que nous noterons $(\Omega, \mathcal{B}, \mathbb{P})$. A tout $f \in L^{2}(\mu)$, on associe la suite gaussienne suivante :

$$
\begin{aligned}
\forall x \in X, \forall \omega \in \Omega, \forall E \subset \mathbb{N}, \sharp(E)<\infty, \\
F_{E, f}(\omega, x)=\frac{1}{\sqrt{\sharp(E)}} \sum_{j \in E} g_{j}(\omega) T_{j}(f)(x) .
\end{aligned}
$$

Lorsque $E=\{1,2, \ldots, J\}$ on notera $F_{E, f}=F_{J, f}$, par abus d'écriture. Le lemme de comparaison qui suit sera crucial pour établir les théorèmes 1.1 et 1.5 .

Lemme 2.3. Soient $S_{n}: L^{2}(\mu) \rightarrow L^{2}(\mu), n=1,2, \ldots$, des opérateurs continus vérifiant $(\mathrm{H} 1)$. Soit $f \in L^{\infty}(\mu)$. Soit I une partie finie de $\mathbb{N}$ telle que

$$
\forall n, m \in I, \quad m \neq n \Leftrightarrow\left\|S_{n}(f)-S_{m}(f)\right\|_{2, \mu} \neq 0 .
$$

Alors, pour tout $0<\varepsilon<1$, de tout index partiel $\mathcal{J}_{0}$, on peut extraire un index partiel $\mathcal{J}$ tel que si

$A(I)=\left\{\forall J \in \mathcal{J}, \forall n, m \in I, m \neq n, \quad \frac{\left\|S_{n}\left(F_{J, f}\right)-S_{m}\left(F_{J, f}\right)\right\|_{2, \mathbb{P}}}{\left\|S_{n}(f)-S_{m}(f)\right\|_{2, \mu}} \geq \sqrt{1-\varepsilon}\right\}$, alors

$$
\mu\{A(I)\} \geq \sqrt{1-\varepsilon},
$$

et pour toute fonction convexe positive croissante $G$ sur $\mathbb{R}^{+}$, 


$$
\begin{array}{r}
\forall J \in \mathcal{J}, \quad \sqrt{1-\varepsilon} \mathbb{E} G\left(\sqrt{1-\varepsilon} \sup _{n, m \in I}\left(Z\left(S_{n}(f)\right)-Z\left(S_{m}(f)\right)\right)\right) \\
\leq \mathbb{E} \int G\left(\sup _{n, m \in I}\left(S_{n}\left(F_{J, f}\right)-S_{m}\left(F_{J, f}\right)\right)\right) d \mu,
\end{array}
$$

où les éléments $F_{J, f}$ sont définis en (2.8), et où $Z$ est le processus gaussien canonique sur $L^{2}(\mu)$. En particulier,

$$
\forall J \in \mathcal{J}, \quad(1-\varepsilon) \mathbb{E} \sup _{n \in I} Z\left(S_{n}(f)\right) \leq \mathbb{E} \int \sup _{n \in I} S_{n}\left(F_{J, f}\right) d \mu .
$$

Démonstration. Nous la produisons pour $\mathcal{J}_{0}=\{1,2, \ldots\}$ seulement, le cas d'un index $\mathcal{J}_{0}$ arbitraire ne présentant aucune difficulté supplémentaire. Soit $0<\varepsilon<1$ fixé. Supposons l'hypothèse (H1) vérifiée, et soit $f \in L^{\infty}(\mu)$. Par hypothèse, les $S_{n}$ commutent avec les $T_{j} ;$ donc $S_{n}\left(F_{J, f}\right)=$ $F_{J, S_{n}(f)}$. Par conséquent,

$$
\left\|S_{n}\left(F_{J, f}\right)-S_{m}\left(F_{J, f}\right)\right\|_{2, \mathbb{P}}^{2}=\frac{1}{J} \sum_{j \leq J}\left[T_{j}\left(S_{n}(f)-S_{m}(f)\right)\right]^{2} .
$$

Le lemme 2.1 et l'hypothèse (H1) nous permettent d'écrire

$$
\begin{gathered}
\left\|S_{n}\left(F_{J, f}\right)-S_{m}\left(F_{J, f}\right)\right\|_{2, \mathbb{P}}^{2}=\frac{1}{J} \sum_{j \leq J} T_{j}\left(S_{n}(f)-S_{m}(f)\right)^{2} \\
\stackrel{L^{1}(\mu)}{\longrightarrow}\left\|S_{n}(f)-S_{m}(f)\right\|_{2, \mu}^{2},
\end{gathered}
$$

quand $J$ tend vers l'infini. Fixons $n, m \in I, n \neq m$. On peut donc déterminer un index partiel $\mathcal{J}_{\star}=\left\{J_{k}: k \geq 1\right\}$, tel que pour tout $k \geq 1$,

$$
\left\|\frac{1}{J_{k}} \sum_{j \leq J_{k}} T_{j}\left(S_{n}(f)-S_{m}(f)\right)^{2}-\right\| S_{n}(f)-S_{m}(f)\left\|_{2, \mu}^{2}\right\|_{1, \mu} \leq 2^{-2 k} .
$$

Donc, pour tout $k \geq 1$,

$$
\mu\left\{\left|\frac{1}{J_{k}} \sum_{j \leq J_{k}} T_{j}\left(S_{n}(f)-S_{m}(f)\right)^{2}-\left\|S_{n}(f)-S_{m}(f)\right\|_{2, \mu}^{2}\right| \geq 2^{-k}\right\} \leq 2^{-k} .
$$

Soit $L \geq 1$ un entier tel que $2^{-L-1} \leq \varepsilon\left\|S_{n}(f)-S_{m}(f)\right\|_{2, \mu}^{2}$. Alors, pour tout $k>L$,

$$
\left\|S_{n}(f)-S_{m}(f)\right\|_{2, \mu}^{2}-2^{-k} \geq(1-\varepsilon)\left\|S_{n}(f)-S_{m}(f)\right\|_{2, \mu}^{2} .
$$

Et par suite,

$$
\mu\left\{\forall k>L, \quad\left\|S_{n}\left(F_{J_{k}, f}\right)-S_{m}\left(F_{J_{k}, f}\right)\right\|_{2, \mathbb{P}} \geq \sqrt{1-\varepsilon}\left\|S_{n}(f)-S_{m}(f)\right\|_{2, \mu}\right\}
$$




$$
\begin{aligned}
& \geq \mu\left\{\forall k>L, \quad\left|\frac{1}{J_{k}} \sum_{j \leq J_{k}} T_{j}\left(S_{n}(f)-S_{m}(f)\right)^{2}-\left\|S_{n}(f)-S_{m}(f)\right\|_{2, \mu}^{2}\right| \leq 2^{-k}\right\} \\
& \geq 1-\sum_{k>L} 2^{-k}=1-2^{-L} .
\end{aligned}
$$

Notons $\mathcal{J}(m, n)=\left\{J_{k}: k>L\right\}$. On a donc montré

$$
\mu\left\{\forall J \in \mathcal{J}(m, n), \quad \frac{\left\|S_{n}\left(F_{J, f}\right)-S_{m}\left(F_{J, f}\right)\right\|_{2, \mathbb{P}}}{\left\|S_{n}(f)-S_{m}(f)\right\|_{2, \mu}} \geq \sqrt{1-\varepsilon}\right\} \geq 1-2^{-L} .
$$

Soit $\left(m^{\prime}, n^{\prime}\right), m^{\prime} \neq n^{\prime}$, un autre couple d'éléments de $I$. Soit aussi $L^{\prime}$ un entier positif suffisamment grand. Puisque

$$
\lim _{\substack{J \rightarrow \infty \\ J \in \mathcal{J}(m, n)}}\left\|\frac{1}{J} \sum_{j \leq J} T_{j}\left(S_{n^{\prime}}(f)-S_{m^{\prime}}(f)\right)^{2}-\right\| S_{n^{\prime}}(f)-S_{m^{\prime}}(f)\left\|_{2, \mu}^{2}\right\|_{1, \mu}=0,
$$

le raisonnement tenu précédemment permet d'extraire de $\mathcal{J}(m, n)$ un autre index partiel $\mathcal{J}\left(m^{\prime}, n^{\prime}\right)$ tel que

$$
\mu\left\{\forall J \in \mathcal{J}\left(m^{\prime}, n^{\prime}\right), \quad \frac{\left\|S_{n^{\prime}}\left(F_{J, f}\right)-S_{m^{\prime}}\left(F_{J, f}\right)\right\|_{2, \mathbb{P}}}{\left\|S_{n^{\prime}}(f)-S_{m^{\prime}}(f)\right\|_{2, \mu}} \geq \sqrt{1-\varepsilon}\right\} \geq 1-2^{-L^{\prime}} .
$$

Procédant alors par extractions successives, on peut déterminer, pour un choix convenable des entiers $L, L^{\prime}, \ldots$, un index partiel $\mathcal{J}=\mathcal{J}(I, \varepsilon)$ tel que si

$A(I)=\left\{\forall J \in \mathcal{J}, \forall n, m \in I, m \neq n, \quad \frac{\left\|S_{n}\left(F_{J, f}\right)-S_{m}\left(F_{J, f}\right)\right\|_{2, \mathbb{P}}}{\left\|S_{n}(f)-S_{m}(f)\right\|_{2, \mu}} \geq \sqrt{1-\varepsilon}\right\}$, alors

$$
\mu\{A(I)\} \geq \sqrt{1-\varepsilon} .
$$

Le long de cet index, on aura donc, en vertu du lemme 2.2,

$$
\begin{aligned}
(1-\varepsilon) \mathbb{E} \sup _{n \in I} Z\left(S_{n}(f)\right) & \leq \sqrt{1-\varepsilon} \int_{A(I)} \mathbb{E} \sup _{n \in I} Z\left(S_{n}(f)\right) d \mu \\
& \leq \int_{A(I)} \mathbb{E} \sup _{n \in I} S_{n}\left(F_{J, f}\right) d \mu \\
& \leq \mathbb{E} \int_{X} \sup _{n \in I} S_{n}\left(F_{J, f}\right) d \mu
\end{aligned}
$$

$\operatorname{car} \mu\left\{\mathbb{E} \sup _{n \in I} S_{n}\left(F_{J, f}\right) \geq 0\right\}=1$. Ceci établit $(2.10 \mathrm{~b})$.

Quant à (2.10a), l'inégalité (2.5) du lemme 2.2 et le fait que $\mu\{A(I)\} \geq$ $\sqrt{1-\varepsilon}$ montrent de même 


$$
\begin{aligned}
\sqrt{1-\varepsilon} \mathbb{E} G\left(\sqrt{1-\varepsilon} \sup _{n, m \in I}\left(Z\left(S_{n}(f)\right)-Z\left(S_{m}(f)\right)\right)\right) \\
\leq \int_{A(I)} \mathbb{E} G\left(\sqrt{1-\varepsilon} \sup _{n, m \in I}\left(Z\left(S_{n}(f)\right)-Z\left(S_{m}(f)\right)\right)\right) d \mu \\
\leq \int_{A(I)} \mathbb{E} G\left(\sup _{n, m \in I} S_{n}\left(F_{J, f}\right)-S_{m}\left(F_{J, f}\right)\right) d \mu \\
\leq \int_{X} \mathbb{E} G\left(\sup _{n, m \in I} S_{n}\left(F_{J, f}\right)-S_{m}\left(F_{J, f}\right)\right) d \mu .
\end{aligned}
$$

Ceci achève donc la démonstration du lemme 2.3.

Enonçons à présent un résultat technique sur les variables aléatoires gaussiennes dû à Komatu-Pollak $[\mathrm{P}]$ :

LEMmE 2.4. Le reste de Mills $R(x)=e^{x^{2} / 2} \int_{x}^{\infty} e^{-t^{2} / 2} d t$ vérifie

$$
\forall x \geq 0, \quad \frac{2}{\sqrt{x^{2}+4}+x} \leq R(x) \leq \frac{2}{\sqrt{x^{2}+8 / \pi}+x} \leq \sqrt{\frac{\pi}{2}} .
$$

En outre, pour toute variable aléatoire $g \stackrel{\mathcal{L}}{=} \mathcal{N}(0,1)$, et tout $T>0$,

$$
\mathbb{E} g^{2} \mathbf{1}_{\{|g| \geq T\}} \leq 2 e^{-T^{2} / 4}
$$

Démonstration. Nous renvoyons à $[\mathrm{P}]$ pour la preuve de (2.11). Quant à (2.12), il suffit d'observer que

$$
\begin{aligned}
\mathbb{E} g^{2} \mathbf{1}_{\{|g| \geq T\}} & =\sqrt{2 / \pi} \int_{T}^{\infty} x^{2} e^{-x^{2} / 2} d x=\sqrt{2 / \pi}\left(T e^{-T^{2} / 2}+\int_{T}^{\infty} e^{-x^{2} / 2} d x\right) \\
& \leq(\sqrt{2 / \pi} T+1) e^{-T^{2} / 2} \leq(T+1) e^{-T^{2} / 2} \leq 2 e^{-T^{2} / 4} .
\end{aligned}
$$

2.3. Les lemmes qui suivent sont relatifs à la démonstration du théorème 1.8. Commençons par établir quelques propriétés utiles d'orthogonalité faible pour des familles d'éléments de $L^{2}(\mu)$. Soit $\left\{T_{j}: j \geq 1\right\}$ une suite d'isométries positives, préservant $1\left(T_{j} 1=1\right.$ pour tout $\left.j\right)$ et faiblement mélangeante :

$$
\forall f, g \in L^{2}(\mu), \quad \lim _{n \rightarrow \infty} \frac{1}{n} \sum_{k=1}^{n}\left|\left\langle T_{k} f, g\right\rangle-\mu(f) \mu(g)\right|=0 .
$$

Etablissons tout d'abord un lemme utile :

LEMme 2.5. Soient $I$ et $J$ deux entiers positifs. Soient $\mathcal{F}=\left\{f_{1}, \ldots, f_{I}\right\}$ et $\mathcal{G}=\left\{g_{1}, \ldots, g_{J}\right\}$ deux sous-ensembles de $L^{2}(\mu) \cap \mathbf{1}_{\mu}^{\perp}$. Alors, pour tout $\varepsilon>0$, on peut trouver un entier $k=k(\varepsilon) \geq 1$ tel que

$$
\sup _{1 \leq i \leq I, 1 \leq j \leq J}\left|\left\langle T_{k} f_{i}, g_{j}\right\rangle\right| \leq \varepsilon
$$

De plus, $k(\varepsilon) \rightarrow \infty$ quand $\varepsilon \rightarrow 0$. 
Démonstration. On peut démontrer ce lemme en invoquant le lemme de Koopmann [K], mais ce n'est pas nécessaire. En effet : reformulons tout d'abord la propriété (2.14) comme suit :

$$
\begin{array}{r}
\forall \varepsilon>0, \exists m_{\varepsilon}<\infty, \forall n \geq m_{\varepsilon}, \exists n \leq k \leq 2 n, \\
\sup _{1 \leq i \leq I, 1 \leq j \leq J}\left|\left\langle T_{k} f_{i}, g_{j}\right\rangle\right| \leq \varepsilon .
\end{array}
$$

Supposons que cette propriété ne soit pas vraie. Alors, il existe un réel $\varepsilon_{0}>0$ et une suite croissante d'entiers positifs $\left(n_{p}\right)_{p \geq 1}$ tels que

$$
\forall p \geq 1, \forall k=n_{p}, \ldots, 2 n_{p}, \quad \sup _{1 \leq i \leq I, 1 \leq j \leq J}\left|\left\langle T_{k} f_{i}, g_{j}\right\rangle\right|>\varepsilon_{0} .
$$

Soit $n_{p}$ fixé ; introduisons les ensembles $I_{i}^{j}\left(n_{p}\right)=\left\{n_{p} \leq k \leq 2 n_{p}:\left|\left\langle T_{k} f_{i}, g_{j}\right\rangle\right|\right.$ $\left.>\varepsilon_{0}\right\}, 1 \leq i \leq I, 1 \leq j \leq J$. Leur réunion recouvre $\left\{n_{p}, n_{p}+1, \ldots, 2 n_{p}\right\}$. Par conséquent, il existe une paire d'indices $\left(i_{p}, j_{p}\right)$ telle que $\sharp\left(I_{i_{p}}^{j_{p}}\left(n_{p}\right)\right) \geq$ $n_{p} /(I \cdot J)$.

Parmi la famille de suites $\left(I_{i}^{j}\left(n_{p}\right)\right)_{p \geq 1}, 1 \leq i \leq J, 1 \leq j \leq J$, il en existe donc au moins une telle que l'inégalité $\sharp\left(I_{i}^{j}\left(n_{p}\right)\right) \geq n_{p} /(I \cdot J)$ ait lieu pour une infinité d'indices $p$. Pour ce choix d'indices $(i, j)$, on a donc

$$
\frac{1}{2 n_{p}} \sum_{k=1}^{2 n_{p}}\left|\left\langle T_{k} f_{i}, g_{j}\right\rangle\right| \geq \frac{\varepsilon_{0}}{2 n_{p}} \sharp\left(I_{i}^{j}\left(n_{p}\right)\right) \geq \frac{\varepsilon_{0}}{2 I \cdot J}
$$

pour une infinité d'indices $p$. Ceci produit une contradiction avec l'hypothèse de mélange faible, puisque l'on sait que

$$
\lim _{n \rightarrow \infty} \frac{1}{n} \sum_{k=1}^{n}\left|\left\langle T_{k} f_{i}, g_{j}\right\rangle\right|=0 .
$$

D'où le lemme.

Nous allons en déduire la proposition suivante :

Proposition 2.6. Soit $\left\{T_{j}: j \geq 1\right\}$ une suite d'isométries positives, préservant 1 et vérifiant la propriété (2.13). Soit $\mathcal{F}=\left\{f_{l}: l \geq 1\right\}$ un sous-ensemble de $L^{2}(\mu) \cap \mathbf{1}_{\mu}^{\perp}$. On suppose que les $f_{l} \neq 0$ sont deux à deux distincts. Soit $\varepsilon=\left\{\varepsilon_{p}: p \geq 1\right\}$ une suite décroissante de réels positifs. Alors, on peut trouver une suite d'entiers $\mathcal{N}=\left\{n_{p}: p \geq 1\right\}$ telle que pour tout p, la propriété $\left(\mathcal{R}_{p}\right)$ ci-dessous soit réalisée :

$$
\sup _{1 \leq k \leq p, 1 \leq i \leq j \leq p}\left|\left\langle T_{n_{p+1}} f_{k}, T_{n_{j}} f_{i}\right\rangle\right| \leq \varepsilon_{p} .
$$

Démonstration. On établit celle-ci par induction en itérant l'argument produit dans le lemme précédent. 
EtAPe $1\left(l=1, n_{1}=1\right)$ : Appliquons le lemme 2.5 au système $\mathcal{F}=\left\{f_{1}\right\}$, $\mathcal{G}=\left\{T_{n_{1}} f_{1}\right\}, \varepsilon=\varepsilon_{1}$. Il existe donc un entier $k=n_{2}>0$ tel que

$$
\left|\left\langle T_{n_{2}} f_{1}, T_{n_{1}} f_{1}\right\rangle\right| \leq \varepsilon_{1} .
$$

Etape 2 (Induction) : Soit $p \geq 1$ fixé. Supposons que la propriété $\left(\mathcal{R}_{p}\right)$ soit vraie. Soit $n_{1}, \ldots, n_{p+1}$ la suite obtenue. Montrons $\left(\mathcal{R}_{p+1}\right)$. Appliquons alors le lemme 2.5 aux systèmes $\mathcal{F}=\left\{f_{1}, \ldots, f_{p+1}\right\}, \mathcal{G}=\left\{T_{n_{1}} f_{1}, \ldots\right.$ $\left.\ldots, T_{n_{p+1}} f_{1}, T_{n_{2}} f_{2}, \ldots, T_{n_{p+1}} f_{2}, \ldots, T_{n_{k}} f_{k}, \ldots, T_{n_{p+1}} f_{k}, \ldots, T_{n_{p+1}} f_{p}\right\}, \varepsilon=$ $\varepsilon_{p+1}$. Il existe donc un entier positif $k=n_{p+2}$ tel que

$$
\sup _{f \in \mathcal{F}, g \in \mathcal{G}}\left|\left\langle T_{n_{p+2}} f, g\right\rangle\right| \leq \varepsilon_{p+1},
$$

c'est-à-dire

$$
\sup _{1 \leq i \leq p+1, g \in \mathcal{G}}\left|\left\langle T_{n_{p+2}} f_{i}, g\right\rangle\right| \leq \varepsilon_{p+1},
$$

ce qui est $\left(\mathcal{R}_{p+1}\right)$. D'où la proposition 2.6.

2.4. Etablissons à présent une inégalité-clé pour la démonstration du théorème 1.8. Soient $\Delta$ une partie finie de $\mathbb{N}$, et $E$ une partie finie de $\mathcal{E}=$ $\left(T_{j}\right)_{j}$. Fixons $x \in X$ et posons

$$
R=R(x)=\sup _{T \in E} \sup _{n \in \Delta}\left|T S_{n} f(x)\right| .
$$

Supposons $R>0$. Il existe donc $m \in \Delta$ et $U \in E$ tels que $\left|U S_{m} f(x)\right|$ $=R$. Soit $(\Omega, \mathcal{B}, \mathbb{P})$ un autre espace probabilisé, sur lequel on considère une suite isonormale $g=\left\{g_{T}: T \in \mathcal{E}\right\}$. Soit aussi $\lambda_{0}>0$ tel que $\mathbb{P}\left\{\left|g_{U}\right| \geq \lambda_{0}\right\} \geq$ 3/4. Si $T S_{m} f(x)=0$ pour tout $T \neq U$, alors pour tout $\omega \in\left\{\left|g_{U}\right| \geq \lambda_{0}\right\}$,

$$
\left|\frac{1}{\sqrt{\sharp(E)}} \sum_{T \in E} g_{T}(\omega) T S_{m} f(x)\right|=\frac{\left|g_{U}(\omega)\right| \cdot\left|U S_{m} f(x)\right|}{\sqrt{\sharp(E)}} \geq \frac{\lambda_{0} R}{\sqrt{\sharp(E)}} .
$$

Sinon, posons

$$
E_{x}=\left\{\omega: \text { signe }\left(\frac{1}{\sqrt{\sharp(E)}} \sum_{\substack{T \in E \\ T \neq U}} g_{T}(\omega) T S_{m} f(x)\right)=\operatorname{signe}\left(g_{U}(\omega) U S_{m} f(x)\right)\right\} .
$$

Par symétrie et indépendance des lois des $g_{T}, \mathbb{P}\left(E_{x}\right)=1 / 2$. Alors si $F_{x}=$ $E_{x} \cap\left\{\left|g_{U}\right| \geq \lambda_{0}\right\}$, on a $\mathbb{P}\left(F_{x}\right) \geq 1 / 4$, et sur $F_{x}$,

$$
\left|\frac{1}{\sqrt{\sharp(E)}} \sum_{T \in E} g_{T}(\omega) T S_{m} f(x)\right| \geq \frac{\left|g_{U}(\omega)\right| \cdot\left|U S_{m} f(x)\right|}{\sqrt{\sharp(E)}} \geq \frac{\lambda_{0} R}{\sqrt{\sharp(E)}} .
$$

Cette inégalité est trivialement vraie si $R=0$. En utilisant la propriété de commutation de $S_{n}$ avec les $T_{j}$, on obtient par conséquent : pour tout $x \in X$, il existe un ensemble $F_{x} \in \sigma\left(g_{T}, T \in E\right)$ tel que $\mathbb{P}\left(F_{x}\right) \geq 1 / 4$ et pour tout $\omega \in F_{x}$, 


$$
\frac{\lambda_{0}}{\sqrt{\sharp E}}\left(\sup _{T \in E} \sup _{n \in \Delta}\left|T S_{n} f(x)\right|\right) \leq \sup _{n \in \Delta}\left|S_{n}\left(\frac{1}{\sqrt{\sharp(E)}} \sum_{T \in E} g_{T} T f(x)\right)\right| .
$$

Tirons-en deux conséquences. D'une part, en intégrant par rapport à $\mu \times \mathbb{P}$ : pour tout $M>0$,

$$
\begin{aligned}
& \mu\left\{\sup _{T \in E} \sup _{n \in \Delta}\left|T S_{n} f(x)\right|>M\right\} \\
& \qquad \leq 4 \mu \times \mathbb{P}\left\{\sup _{n \in \Delta}\left|S_{n}\left(\frac{1}{\sqrt{\sharp(E)}} \sum_{T \in E} g_{T} T f(x)\right)\right|>\frac{M \lambda_{0}}{\sqrt{\sharp E}}\right\} .
\end{aligned}
$$

Soit d'autre part $B \in \mathcal{A}$; en intégrant par rapport à $\mathbb{P}$ sur $F_{x}$, puis par rapport à $\mu$ sur $B$, et en utilisant le théorème de Fubini, il vient

$$
\begin{aligned}
\iint_{B} \sup _{n \in \Delta}\left|S_{n}\left(\frac{1}{\sqrt{\sharp(E)}} \sum_{T \in E} g_{T} T f(x)\right)\right| d \mathbb{P} d \mu & \\
& \geq \frac{\lambda_{0}}{4 \sqrt{\sharp(E)}}\left(\int_{B} \sup _{T \in E} \sup _{n \in \Delta}\left|T S_{n} f\right| d \mu\right) .
\end{aligned}
$$

Par conséquent, on a

Lemme 2.7. Pour tous sous-ensembles finis $\Delta \subset \mathbb{N}$ et $E \subset\left(T_{j}\right)_{j}$, et tout $x \in X$,

$$
\mathbb{P}\left\{\sup _{n \in \Delta}\left|S_{n}\left(\frac{1}{\sqrt{\sharp(E)}} \sum_{T \in E} g_{T} T f(x)\right)\right| \geq \frac{\lambda_{0}}{\sqrt{\sharp E}}\left(\sup _{T \in E} \sup _{n \in \Delta}\left|T S_{n} f(x)\right|\right)\right\} \geq \frac{1}{4} .
$$

En outre,

(a) pour tout $M>0$,

$$
\begin{aligned}
\mu\left\{\sup _{T \in E} \sup _{n \in \Delta}\left|T S_{n} f\right|\right. & >M\} \\
& \leq 4 \mu \times \mathbb{P}\left\{\sup _{n \in \Delta}\left|S_{n}\left(\frac{1}{\sqrt{\sharp(E)}} \sum_{T \in E} g_{T} T f\right)\right|>\frac{M \lambda_{0}}{\sqrt{\sharp E}}\right\},
\end{aligned}
$$

(b) pour tout $B \in \mathcal{A}$,

$$
\frac{\lambda_{0}}{\sqrt{\sharp(E)}} \int_{B} \sup _{T \in E} \sup _{n \in \Delta}\left|T S_{n} f\right| d \mu \leq \int_{B} \int_{\Omega} \sup _{n \in \Delta}\left|S_{n}\left(\frac{1}{\sqrt{\sharp(E)}} \sum_{T \in E} g_{T} T f\right)\right| d \mathbb{P} d \mu \text {. }
$$

2.5. Rappelons ici l'extension par Bellow-Jones du principe de Banach dans $L^{\infty}(\mu)$. Notons

$$
\mathcal{Y}=\left\{f \in L^{\infty}(\mu):\|f\|_{\infty} \leq 1\right\}
$$

On munit $\mathcal{Y}$ de la distance $d$ associée à la convergence en mesure. Observons que les distances $d$ et $\|\cdot\|_{p}, 1 \leq p<\infty$, sont équivalentes sur $\mathcal{Y}$. Soit $S: \mathcal{Y} \rightarrow L^{0}(\mu)$ non nécessairement linéaire. On dit que $S$ est continu en 0 si $S$ est $d$-continu en 0 sur $\mathcal{Y}$. 
Proposition 2.8 ([BJ], Théorème 2 ). Soit $\left\{S_{n}: n \geq 1\right\}$ une suite d'opérateurs linéaires de $L^{\infty}(\mu)$ dans $L^{0}(\mu)$. Supposons que les conditions suivantes soient réalisées:

(a) la suite $\left\{S_{n}: n \geq 1\right\}$ est équicontinue en mesure:

$\forall \delta>0, \exists \eta=\eta(\delta)>0 \forall f, g \in L^{\infty}(\mu)$,

$$
d(f, g)<\eta \Rightarrow \forall n \geq 1, d\left(S_{n}(f), S_{n}(g)\right) \leq \delta .
$$

(b) $\forall f \in L^{\infty}(\mu), \mu\left\{\left\{S_{n}(f): n \geq 1\right\}\right.$ converge $\}=1$.

Alors $S^{*}: \mathcal{Y} \rightarrow L^{0}(\mu)$ est continu en 0 .

3. Démonstration du théorème 1.1. Il suffit d'établir le résultat pour les parties finies de $\mathbb{N}$. Soit $I$ une partie finie de $\mathbb{N}$. Montrons l'inégalité de droite. Observons tout d'abord que

$$
(\mathrm{HM}) \Rightarrow \Omega_{1}(S, I) \leq \Omega_{2}(S, I) \leq C .
$$

Mais

$$
\begin{aligned}
\mathbb{E} \sup _{n \in I}\left|Z\left(S_{n}(f)\right)\right| & \geq \sup _{n \in I} \mathbb{E}\left|Z\left(S_{n}(f)\right)\right|=\sqrt{2 / \pi} \sup _{n \in I} \mathbb{E}\left(\left|Z\left(S_{n}(f)\right)\right|^{2}\right)^{1 / 2} \\
& =\sqrt{2 / \pi} \sup _{n \in I}\left\|S_{n}(f)\right\|_{2, \mu} .
\end{aligned}
$$

Donc

$$
\mathbf{\Phi}_{a}(S, I) \geq \sqrt{2 / \pi} \sup _{n \in I}\left\|S_{n}\right\|
$$

ce qui établit

$$
\boldsymbol{\Omega}_{2}(S, I) \leq \sqrt{\frac{\pi}{2}} C \frac{\boldsymbol{\Phi}_{a}(S, I)}{S(I, 2)} .
$$

L'inégalité de gauche résulte de la proposition suivante :

Proposition 3.1. Soient $S_{n}: L^{2}(\mu) \rightarrow L^{2}(\mu), n=1,2, \ldots$, des opérateurs continus vérifiant l'hypothèse $(\mathrm{H} 1)$. Soit I une partie finie de $\mathbb{N}$; alors

$$
\mathbf{\Phi}(S, I) \leq \boldsymbol{\Omega}_{1}(S, I) .
$$

Démonstration. Soit $f \in L^{\infty}(\mu)$ telle que $\|f\|_{2, \mu}=1$. En vertu du Lemme 2.3, pour tout $0<\varepsilon<1$ il existe un index partiel $\mathcal{J}$ tel que

$$
\forall J \in \mathcal{J}, \quad(1-\varepsilon) \mathbb{E} \sup _{n \in I} Z\left(S_{n}(f)\right) \leq \mathbb{E} \int \sup _{n \in I} S_{n}\left(F_{J, f}\right) d \mu,
$$

où les éléments $F_{J, f}$ sont définis en (2.8), et où $Z$ est le processus gaussien canonique sur $L^{2}(\mu)$. Soit $0<\varepsilon<1$ et posons $u_{0}=0, u_{n}=\varepsilon(1+\varepsilon)^{n-1}$, $n=1,2 \ldots$ Ecrivons que 


$$
\begin{aligned}
\mathbb{E} \int \sup _{n \in I} \mid & S_{n}\left(F_{J, f}\right) \mid d \mu \\
& =\sum_{k=1}^{\infty} \mathbb{E}\left(\mathbf{1}_{\left\{u_{k-1} \leq\left\|F_{J, f}\right\|_{2, \mu}<u_{k}\right\}} \cdot \int \sup _{n \in I}\left|S_{n}\left(F_{J, f}\right)\right| d \mu\right) \\
& \leq \sum_{k=1}^{\infty} \mathbb{P}\left\{u_{k-1} \leq\left\|F_{J, f}\right\|_{2, \mu}<u_{k}\right\} \sup _{u_{k-1} \leq\|g\|_{2, \mu}<u_{k}} \int_{n \in I} \sup _{n \in I}\left|S_{n}(g)\right| d \mu \\
& \leq \boldsymbol{\Omega}_{1}(S, I) \sum_{k=1}^{\infty} u_{k} \mathbb{P}\left\{u_{k-1} \leq\left\|F_{J, f}\right\|_{2, \mu}<u_{k}\right\} \\
& \leq \boldsymbol{\Omega}_{1}(S, I)\left(u_{1} \mathbb{P}\left\{\left\|F_{J, f}\right\|_{2, \mu}<u_{1}\right\}+(1+\varepsilon) \mathbb{E}\left\|F_{J, f}\right\|_{2, \mu} \cdot \mathbf{1}_{u_{1} \leq\left\|F_{J, f}\right\|_{2, \mu}}\right) \\
& \leq \mathbf{\Omega}_{1}(S, I)\left(\varepsilon+(1+\varepsilon) \mathbb{E}\left\|F_{J, f}\right\|_{2, \mu}\right) \leq \mathbf{\Omega}_{1}(S, I)(1+2 \varepsilon),
\end{aligned}
$$

cette dernière inégalité provenant de l'estimation ci-dessous :

$$
\mathbb{E}\left\|F_{J, f}\right\|_{2, \mu} \leq\left[\int_{X} \mathbb{E}\left|\frac{1}{\sqrt{J}} \sum_{j \leq J} g_{j} T_{j} f\right|^{2} d \mu\right]^{1 / 2} \leq\left[\int_{X} \frac{1}{J} \sum_{j \leq J}\left(T_{j} f\right)^{2} d \mu\right]^{1 / 2} \leq 1 .
$$

Finalement, en recollant ces estimations et en faisant tendre $\varepsilon$ vers $0+$, il vient

$$
\mathbb{E} \sup _{n \in I} Z\left(S_{n}(f)\right) \leq \boldsymbol{\Omega}_{1}(S, I) .
$$

Soit à présent $f \in L^{2}(\mu)$ telle que $\|f\|_{2, \mu}=1$, et soit $\left(f_{k}\right)_{k}$ une suite d'éléments de $L^{\infty}(\mu)$ approchant $f$ dans $L^{2}(\mu)$. Comme

$$
\lim _{k \rightarrow \infty} \mathbb{E} \sup _{n \in I} Z\left(S_{n}\left(f_{k}\right)\right)=\mathbb{E} \sup _{n \in I} Z\left(S_{n}(f)\right),
$$

on en déduit (3.2) dans ce cas aussi.

Montrons maintenant comment achever la démonstration du théorème 1.1. Soit $f \in L^{2}(\mu)$ telle que $\|f\|_{2, \mu}=1$. Observons que pour tout $m \in I$,

$$
\begin{aligned}
\mathbb{E} \sup _{n \in I}\left|Z\left(S_{n}(f)\right)\right| & \leq \mathbb{E}\left|Z\left(S_{m}(f)\right)\right|+\mathbb{E} \sup _{n \in I}\left|Z\left(S_{n}(f)\right)-Z\left(S_{m}(f)\right)\right| \\
& =\sqrt{2 / \pi}\left\|Z\left(S_{m}(f)\right)\right\|_{2}+\mathbb{E} \sup _{n \in I}\left(Z\left(S_{n}(f)\right)-Z\left(S_{m}(f)\right)\right) \\
& =\sqrt{2 / \pi}\left\|Z\left(S_{m}(f)\right)\right\|_{2}+\mathbb{E} \sup _{n \in I} Z\left(S_{n}(f)\right) \\
& \leq \sqrt{2 / \pi}\left\|Z\left(S_{m}(f)\right)\right\|_{2}+\mathbf{\Omega}_{1}(S, I) .
\end{aligned}
$$

Et puisque $1+\sqrt{2 / \pi} \leq 9 / 5 \leq 2$,

$$
\mathbb{E} \sup _{n \in I}\left|Z\left(S_{n}(f)\right)\right| \leq \boldsymbol{\Omega}_{1}(S, I)+\sqrt{2 / \pi}\left\|S_{m}\right\| \leq 2 \boldsymbol{\Omega}_{2}(S, I),
$$


car $\boldsymbol{\Omega}_{1}(S, I) \leq \boldsymbol{\Omega}_{2}(S, I)$ et $\left\|S_{m}\right\| \leq \boldsymbol{\Omega}_{2}(S, I)$. On a donc $\mathbb{E} \sup _{n \in I}\left|Z\left(S_{n}(f)\right)\right|$ $\leq 2 \boldsymbol{\Omega}_{1}(S, I)$. D'où $\boldsymbol{\Phi}_{a}(S, I) \leq 2 \boldsymbol{\Omega}_{2}(S, I)$.

4. Démonstration du théorème 1.5. Soit $f \in L^{\infty}(\mu)$ telle que $\|f\|_{2}$ $=1$. Soit $g_{1}, g_{2}, \ldots$ une suite isonormale réalisée sur un espace d'épreuves différent, noté $(\Omega, \mathcal{B}, \mathbb{P})$. Soit $I$ un ensemble fini d'entiers fixé. Associons à $f$ la suite d'éléments de Stein définie en (2.8). Puisque les $T_{j}$ sont des isométries de $L^{2}(\mu)$,

$$
\mathbb{E} \int\left|F_{J, f}\right|^{2} d \mu=\int \frac{1}{J} \sum_{j \leq J}\left[T_{j}(f)\right]^{2} d \mu=1 .
$$

En outre, on a aussi

$$
\begin{aligned}
\mathbb{E} \int \sup _{n \in I}\left|S_{n}\left(F_{J, f}\right)\right|^{2} d \mu & \leq \mathbb{E} \sum_{n \in I} \int\left|S_{n}\left(F_{J, f}\right)\right|^{2} d \mu \\
& \leq \sharp(I) S(I, 2)^{2} \mathbb{E} \int\left|F_{J, f}\right|^{2} d \mu=\sharp(I) S(I, 2)^{2} .
\end{aligned}
$$

Soit $0<\gamma<1$ fixé. En vertu du lemme 2.3, il existe un index partiel $\mathcal{J}$ tel que

$$
\forall J \in \mathcal{J}, \quad \gamma \mathbb{E} \sup _{n \in I} Z\left(S_{n}(f)\right) \leq \mathbb{E} \int \sup _{n \in I} S_{n}\left(F_{J, f}\right) d \mu,
$$

où $Z$ est le processus gaussien canonique sur $L^{2}(\mu)$. Afin d'alléger l'écriture, nous allons noter dans ce qui suit $F_{J}=F_{J, f}$. Posons aussi pour tout $A>0$, $E_{A}=\left\{(\omega, x) \in \Omega \times X:\left|F_{J}(\omega, x)\right| \leq A\right\}, \quad E_{A, \omega}=\left\{x \in X:(\omega, x) \in E_{A}\right\}$, et pour tout $(\omega, x) \in \Omega \times X$,

$$
\begin{aligned}
& F_{A, J}(x)=F_{A, J, \omega}(x)=F_{J}(\omega, x) \cdot \mathbf{1}_{E_{A, \omega}}(x), \\
& F^{A, J}(x)=F^{A, J, \omega}(x)=F_{J}(\omega, x) \cdot \mathbf{1}_{E_{A, \omega}^{c}}(x) .
\end{aligned}
$$

Il existe donc un index partiel $\mathcal{J}$ tel que pour tout $J \in \mathcal{J}$,

$$
\begin{aligned}
\gamma \mathbb{E} \sup _{n \in I} Z\left(S_{n}(f)\right) & \leq \mathbb{E} \int \sup _{n \in I} S_{n}\left(F_{J, f}\right) d \mu \\
& \leq \mathbb{E} \int \sup _{n \in I}\left|S_{n}\left(F^{A, J}\right)\right| d \mu+\mathbb{E} \int \sup _{n \in I}\left|S_{n}\left(F_{A, J}\right)\right| d \mu .
\end{aligned}
$$

Par définition $F_{A, J, \omega}(\cdot)\left(\right.$ resp. $\left.F^{A, J, \omega}\right)$ est $\mathcal{A}$-mesurable. Comme $f \in L^{\infty}(\mu)$, on a

$$
P\left\{\omega: F_{A, J, \omega}(x)\left(\operatorname{resp} . F^{A, J, \omega}\right) \in L^{\infty}(\mu)\right\}=1 .
$$

Par ailleurs, pour presque tout $\omega$,

$$
\begin{aligned}
\int \sup _{n \in I}\left|S_{n}\left(F^{A, J, \omega}\right)\right| d \mu & \leq\left[\sum_{n \in I} \int\left|S_{n}\left(F^{A, J, \omega}\right)\right|^{2} d \mu\right]^{1 / 2} \\
& \leq \sqrt{\sharp(I)} S(I, 2)\left\|F^{A, J, \omega}\right\|_{2, \mu} .
\end{aligned}
$$


Il nous faut donc estimer $\left\|F^{A, J}\right\|_{2, \mu}$. A l'aide du théorème de Fubini, remarquons tout d'abord que

$$
\mathbb{E}\left\|F^{A, J}\right\|_{2, \mu}^{2}=\mathbb{E} \int\left|F_{J, f}\right|^{2} \mathbf{1}_{\left\{\left|F_{J, f}\right| \geq A\right\}} d \mu=\int \mathbb{E}\left|F_{J, f}\right|^{2} \cdot \mathbf{1}_{\left\{\left|F_{J, f}\right| \geq A\right\}} d \mu .
$$

Appliquons alors le lemme 2.4 à $g=F_{J, f} /\left\|F_{J, f}\right\|_{2, \mathbb{P}}$ et $T=A /\left\|F_{J, f}\right\|_{2, \mathbb{P}}$. Nous obtenons

$$
\mathbb{E}\left|F_{J, f}\right|^{2} \cdot \mathbf{1}_{\left\{\left|F_{J, f}\right| \geq A\right\}} \leq 2\left\|F_{J, f}\right\|_{2, \mathbb{P}}^{2} \exp \left(-\frac{A^{2}}{4\left\|F_{J, f}\right\|_{2, \mathbb{P}}^{2}}\right),
$$

$\mu$-presque partout. En intégrant cette dernière estimation relativement à $\mu$, nous arrivons à

$$
\mathbb{E}\left\|F^{A, J}\right\|_{2, \mu}^{2} \leq 2 \int_{X}\left\|F_{J, f}\right\|_{2, \mathbb{P}}^{2} \exp \left(-\frac{A^{2}}{4\left\|F_{J, f}\right\|_{2, \mathbb{P}}^{2}}\right) d \mu .
$$

Mais le fait que $f \in L^{\infty}(\mu),\|f\|_{2, \mu}=1$ et le lemme 2.1 , ainsi que l'hypothèse (H1), impliquent

$$
\left\|F_{J, f}\right\|_{2, \mathbb{P}}^{2}=\frac{1}{J} \sum_{j \leq J} T_{j}\left(f^{2}\right) \stackrel{L^{1}(\mu)}{\longrightarrow} 1,
$$

quand $J$ tend vers l'infini. Quitte à extraire de $\mathcal{J}$ un autre index partiel, que nous notons encore $\mathcal{J}$, nous pouvons écrire

$$
\mu\left\{\lim _{\substack{J \in \mathcal{J} \\ j}} \frac{1}{J} \sum_{j \leq J} T_{j}\left(f^{2}\right)=1\right\}=1 .
$$

Puisque $\left\|F_{J, f}\right\|_{2, \mathbb{P}}^{2} \leq \max _{j}\left\|T_{j}\left(f^{2}\right)\right\|_{\infty} \leq\left\|f^{2}\right\|_{\infty}<\infty$, le théorème de convergence dominée montre donc que l'intégrale du membre à droite de l'estimation (4.7) converge vers $2 e^{-A^{2} / 4}$, quand $J$ tend vers l'infini suivant $\mathcal{J}$. Soit $\alpha>1$ fixé. Quitte à extraire encore, nous avons donc obtenu

$$
\forall J \in \mathcal{J}, \quad \mathbb{E}\left\|F^{A, J}\right\|_{2, \mu}^{2} \leq(1+\alpha) e^{-A^{2} / 4} .
$$

Intégrant alors l'inégalité (4.5) par rapport à $\mathbb{P}$, nous déduisons de (4.4) et (4.9) que pour tout $J \in \mathcal{J}$,

$$
\begin{aligned}
\gamma \mathbb{E} \sup _{n \in I} Z\left(S_{n}(f)\right) \leq & \sqrt{(1+\alpha) \sharp(I)} S(I, 2) e^{-A^{2} / 8} \\
& +\mathbb{E} \int \sup _{n \in I}\left|S_{n}\left(F_{A, J}\right)\right| d \mu .
\end{aligned}
$$

L'estimation de la quantité $\mathbb{E} \int \sup _{n \in I}\left|S_{n}\left(F_{A, J}\right)\right| d \mu$ va résulter d'une évaluation fine de $\mathbb{E} \exp \left(a\left\|F_{A, J}\right\|_{2, \mu}^{2}\right)$, où $a=1 /(4 \alpha)$. Tout d'abord,

$$
\mathbb{E} \exp \left(a\left\|F_{A, J}\right\|_{2, \mu}^{2}\right)=\mathbb{E} \exp \left(a \int_{X} F_{A, J}^{2} d \mu\right)
$$


et, à l'aide de l'inégalité de Jensen,

$$
\leq \mathbb{E} \int_{X} \exp \left(a F_{A, J}^{2}\right) d \mu \leq \mathbb{E} \int_{B_{\alpha}} \exp \left(a F_{A, J}^{2}\right) d \mu+e^{a A^{2}} \mathbb{P}\left(B_{\alpha}^{\mathrm{c}}\right),
$$

où l'ensemble $B_{\alpha}$ sera déterminé plus loin. Or $(1 / J) \sum_{j \leq J} T_{j} f^{2}$ converge dans $L^{1}(\mu)$ vers $\int f^{2} d \mu=1$ quand $J$ tend vers l'infini. Soit $\delta_{k}=\delta 2^{-k}$, $k \geq 1$, où $0<\delta<\inf (\alpha-1,1)$ sera déterminé plus tard. On peut donc extraire de l'index $\mathcal{J}$ une suite $\left(J_{k}\right)$ telle que

$$
\mu\left\{\left|\frac{1}{J_{k}} \sum_{j \leq J_{k}} T_{j} f^{2}-1\right|>\delta_{k}\right\} \leq \delta_{k} .
$$

Posons

$$
\check{B}_{\delta}=\left\{\forall k \geq 1,\left|\frac{1}{J_{k}} \sum_{j \leq J_{k}} T_{j} f^{2}-1\right| \leq \delta_{k}\right\} .
$$

On a $\mu\left(\check{B}_{\delta}\right) \geq 1-\sum_{k=1}^{\infty} \delta_{k}=1-\delta$, et

$$
\check{B}_{\delta} \subset B_{\alpha}:=\left\{\forall k \geq 1, \frac{1}{J_{k}} \sum_{j \leq J_{k}} T_{j} f^{2}<\alpha\right\} .
$$

Nous avons donc $\mu\left(B_{\alpha}\right) \geq 1-\delta$. Et $\operatorname{sur} B_{\alpha}$,

$$
1-2 a\left(\frac{1}{J_{k}} \sum_{j \leq J_{k}} T_{j} f^{2}\right)>1-2 a \alpha=\frac{1}{2},
$$

pour tout $k \geq 1$. Donc,

$$
\int_{B_{\alpha}} \frac{d \mu}{\sqrt{1-2 a\left(1 / J_{k}\right) \sum_{j \leq J_{k}} T_{j} f^{2}}} \leq \sqrt{2} .
$$

Comme $\mathbb{E} \exp b\left(\mathcal{N}(0,1)^{2}\right)=1 / \sqrt{1-2 b}$ pour tout $0 \leq b<1 / 2$, nous avons l'estimation suivante :

$$
\mathbb{E} \exp \left(a\left\|F_{A, J_{k}}\right\|_{2, \mu}^{2}\right) \leq \int_{B_{\alpha}} \frac{d \mu}{\sqrt{1-2 a\left(1 / J_{k}\right) \sum_{j \leq J_{k}} T_{j} f^{2}}}+e^{a A^{2}} \mathbb{P}\left(B_{\alpha}^{\mathrm{c}}\right) .
$$

Donc

$$
\mathbb{E} \exp \left(a\left\|F_{A, J_{k}}\right\|_{2, \mu}^{2}\right) \leq \sqrt{2}+\delta e^{A^{2} a} .
$$

La suite extraite $\left(J_{k}\right)$ dépend de $\delta$. Nous allons choisir $\delta<(\alpha-1) e^{-A^{2} /(4 \alpha)}$. Notons encore par $\mathcal{J}$ la suite $\left(J_{k}\right)$. Alors $\mathcal{J}$ dépend de $A$ et de $\alpha$; et pour tout $J$ élément de cet index, nous avons obtenu l'estimation suivante :

$$
\mathbb{E} \exp \left(a\left\|F_{A, J}\right\|_{2, \mu}^{2}\right) \leq \sqrt{2}+\alpha-1 .
$$


Evaluons à présent la quantité $\mathbb{E} \int \sup _{n \in I}\left|S_{n}\left(F_{A, J}\right)\right| d \mu$ à partir des deux intégrales

$$
\mathbb{E} \int_{B_{\alpha}} \sup _{n \in I}\left|S_{n}\left(F_{A, J}\right)\right| d \mu \quad \text { et } \quad \mathbb{E} \int_{B_{\alpha}^{\mathrm{c}}} \sup _{n \in I}\left|S_{n}\left(F_{A, J}\right)\right| d \mu .
$$

Le premier terme se majore pour tout $R>0$ par

$$
\begin{aligned}
\int_{B_{\alpha}} \mathbb{E}\left(\sup _{n \in I}\left|S_{n}\left(F_{A, J}\right)\right| \mathbf{1}_{\left\{\left\|F_{A, J}\right\|_{2, \mu}>R\right\}}\right) d \mu \\
\\
+\int_{B_{\alpha}} \mathbb{E}\left(\sup _{n \in I}\left|S_{n}\left(F_{A, J}\right)\right| \mathbf{1}_{\left\{\left\|F_{A, J}\right\|_{2, \mu} \leq R\right\}}\right) d \mu .
\end{aligned}
$$

Le second terme se majore comme suit, à l'aide de l'inégalité de CauchySchwarz :

$$
\begin{aligned}
\mathbb{E} \int_{B_{\alpha}^{\mathrm{c}}} \sup _{n \in I}\left|S_{n}\left(F_{A, J}\right)\right| d \mu & \leq \mu\left(B_{\alpha}^{\mathrm{c}}\right)^{1 / 2} \mathbb{E}\left\|\sup _{n \in I}\left|S_{n}\left(F_{A, J}\right)\right|\right\|_{2, \mu} \\
& \leq(\alpha-1)^{1 / 2} e^{-A^{2} /(8 \alpha)} \sqrt{\sharp(I)} S(I, 2) \mathbb{E}\left\|F_{A, J}\right\|_{2, \mu} \\
& \leq(\alpha-1)^{1 / 2} e^{-A^{2} /(8 \alpha)} \sqrt{\sharp(I)} S(I, 2) .
\end{aligned}
$$

Revenons à la majoration du premier terme. Observons que

$$
\left\|S_{n}\left(F_{A, J}\right)\right\|_{\infty} \leq S(I, \infty)\left\|F_{A, J}\right\|_{\infty} \leq S(I, \infty) A .
$$

L'estimation (4.11) et le fait que les $S_{n}$ soient continus sur $L^{\infty}(\mu)$ permettent alors de majorer

$$
\int_{B_{\alpha}} \mathbb{E}\left(\sup _{n \in I}\left|S_{n}\left(F_{A, J}\right)\right| \mathbf{1}_{\left\{\left\|F_{A, J}\right\|_{2, \mu}>R\right\}}\right) d \mu
$$

$\operatorname{par}(\sqrt{2}+\alpha-1) A S(I, \infty) e^{-a R^{2}}$. Occupons-nous de la seconde intégrale. Ici, c'est beaucoup plus facile, car on a la majoration directe

$$
\int_{B_{\alpha}} \mathbb{E}\left(\sup _{n \in I}\left|S_{n}\left(F_{A, J}\right)\right| \mathbf{1}_{\left\{\left\|F_{A, J}\right\|_{2, \mu} \leq R\right\}}\right) d \mu \leq A \boldsymbol{\Omega}_{\infty, 2}(S, I, R / A) .
$$

En recollant toutes ces estimations, et en revenant sur l'inégalité de départ, nous voyons que nous sommes arrivés à

$$
\begin{aligned}
\gamma \mathbb{E} \sup _{n \in I} Z\left(S_{n}(f)\right) \leq & S(I, 2) \sqrt{(1+\alpha) \sharp(I)} e^{-A^{2} / 8} \\
& +S(I, 2)(\alpha-1)^{1 / 2} e^{-A^{2} /(8 \alpha)} \sqrt{\sharp(I)} \\
& +(\sqrt{2}+\alpha-1) A S(I, \infty) e^{-a R^{2}} \\
& +A \boldsymbol{\Omega}_{\infty, 2}(S, I, R / A),
\end{aligned}
$$

écriture dans laquelle $J$ a miraculeusement disparu. Nous étions libres de choisir $\alpha>1$, mais aussi proche de 1 que l'on veut; ce que nous faisons donc 
à présent. En faisant tendre aussi $\gamma$ vers 1 , nous avons ainsi obtenu

$$
\begin{aligned}
\mathbb{E} \sup _{n \in I} Z\left(S_{n}(f)\right) \leq & \sqrt{2 \sharp(I)} S(I, 2) e^{-A^{2} / 8}+\sqrt{2} A S(I, \infty) e^{-R^{2} / 4} \\
& +A \boldsymbol{\Omega}_{\infty, 2}(S, I, R / A) .
\end{aligned}
$$

Cette dernière inégalité étant vérifiée pour tout $f \in L^{\infty}(\mu)$ telle que $\|f\|_{2, \mu}$ $=1$, on en déduit sans peine le résultat annoncé par continuité en moyenne quadratique de $Z$.

REMARque. Le fait que les $S_{n}$ soient continus sur $L^{\infty}(\mu)$ n'est intervenu que dans le contrôle de la quantité

$$
\int_{B_{\alpha}} \mathbb{E}\left(\sup _{n \in I}\left|S_{n}\left(F_{A, J}\right)\right| \mathbf{1}_{\left\{\left\|F_{A, J}\right\|_{2, \mu}>R\right\}}\right) d \mu .
$$

En l'absence de cette hypothèse, on peut malgré tout la majorer à l'aide du théorème de Fubini, puis des inégalités de Hölder et Jensen, et enfin de (4.11) comme suit :

$$
\begin{aligned}
\int_{B_{\alpha}} \mathbb{E}\left[\sup _{n \in I}\left|S_{n}\left(F_{A, J}\right)\right| \mathbf{1}_{\left\{\left\|F_{A, J}\right\|_{2, \mu}>R\right\}}\right] d \mu \\
\quad=\mathbb{E}\left[\left(\int_{B_{\alpha}} \sup _{n \in I}\left|S_{n}\left(F_{A, J}\right)\right| d \mu\right) \cdot \mathbf{1}_{\left\{\left\|F_{A, J}\right\|_{2, \mu}>R\right\}}\right] \\
\leq \mathbb{E}\left[\left\|\sup _{n \in I}\left|S_{n}\left(F_{A, J}\right)\right|\right\|_{2, \mu} \cdot \mathbf{1}_{\left\{\left\|F_{A, J}\right\|_{2, \mu}>R\right\}}\right] \\
\leq S(I, 2) \sqrt{\sharp(I)} \mathbb{E}\left\|F_{A, J}\right\|_{2, \mu} \cdot \mathbf{1}_{\left\{\left\|F_{A, J}\right\|_{2, \mu}>R\right\}} .
\end{aligned}
$$

Mais

$$
\begin{aligned}
\mathbb{E}\left\|F_{A, J}\right\|_{2, \mu} \cdot \mathbf{1}_{\left\{\left\|F_{A, J}\right\|_{2, \mu}>R\right\}} & \\
& =R \mathbb{P}\left\{\left\|F_{A, J}\right\|_{2, \mu}>R\right\}+\int_{R}^{\infty} \mathbb{P}\left\{\left\|F_{A, J}\right\|_{2, \mu}>u\right\} d u .
\end{aligned}
$$

Alors, d'une part à l'aide du lemme 2.4,

$$
\begin{aligned}
\int_{R}^{\infty} \mathbb{P}\left\{\left\|F_{A, J}\right\|_{2, \mu}>u\right\} d u & =\int_{R}^{\infty} \mathbb{P}\left\{\exp \left[a\left\|F_{A, J}\right\|_{2, \mu}^{2}\right] \geq \exp \left[a u^{2}\right]\right\} d u \\
& \leq(\sqrt{2}+\alpha-1) \int_{R}^{\infty} e^{-a u^{2}} d u \\
& \leq(\sqrt{2}+\alpha-1) \sqrt{\pi \alpha} e^{-R^{2} /(4 \alpha)}
\end{aligned}
$$

et d'autre part, en invoquant l'estimation (4.11),

$$
R \mathbb{P}\left\{\left\|F_{A, J}\right\|_{2, \mu}>R\right\} \leq(\sqrt{2}+\alpha-1) R e^{-R^{2} /(4 \alpha)},
$$


et donc

$$
\mathbb{E}\left\|F_{A, J}\right\|_{2, \mu} \cdot \mathbf{1}_{\left\{\left\|F_{A, J}\right\|_{2, \mu}>R\right\}} \leq(\sqrt{2}+\alpha-1)(R+\sqrt{\pi \alpha}) e^{-R^{2} /(4 \alpha)} .
$$

D'où

$$
\begin{aligned}
\int_{B_{\alpha}} \mathbb{E}\left(\sup _{n \in I}\left|S_{n}\left(F_{A, J}\right)\right|\right. & \left.\mathbf{1}_{\left\{\left\|F_{A, J}\right\|_{2, \mu}>R\right\}}\right) d \mu \\
\leq & (\sqrt{2}+\alpha-1)(R+\sqrt{\pi \alpha}) S(I, 2) \sqrt{\sharp(I)} e^{-R^{2} /(4 \alpha)},
\end{aligned}
$$

et finalement

$$
\begin{aligned}
\left(4.12^{\prime}\right) \quad \gamma \mathbb{E} \sup _{n \in I} Z\left(S_{n}(f)\right) \\
\leq S(I, 2) \sqrt{(1+\alpha) \sharp(I)} e^{-A^{2} / 8} \\
+S(I, 2) \sqrt{\alpha-1} e^{-A^{2} /(8 \alpha)} \sqrt{\sharp(I)} \\
+(\sqrt{2}+\alpha-1)(R+\sqrt{\pi \alpha}) S(I, 2) \sqrt{\sharp(I)} e^{-R^{2} /(4 \alpha)} \\
+A \boldsymbol{\Omega}_{\infty, 2}(S, I, R / A) .
\end{aligned}
$$

L'inégalité (4.13) devient alors

$$
\begin{aligned}
\mathbb{E} \sup _{n \in I} Z\left(S_{n}(f)\right) \leq & \sqrt{2 \sharp(I)} S(I, 2) e^{-A^{2} / 8} \\
& +\sqrt{2}(\sqrt{\pi}+R) S(I, 2) \sqrt{\sharp(I)} e^{-R^{2} / 4} \\
& +A \boldsymbol{\Omega}_{\infty, 2}(S, I, R / A) .
\end{aligned}
$$

5. Démonstration du théorème 1.8. Notons $S_{0}=0$. Soit $\Delta$ une partie finie non vide de $\mathbb{N}$. Soient $f \in L^{2}(\mu)$ et $E \subset \mathcal{E}$ de cardinal $q \geq 1$. D'après le lemme 2.7, on a

$$
\forall t>0, \quad \mu\left\{\sup _{T \in E} T S_{\Delta}(f)>t\right\} \leq 4 \mu \times \mathbb{P}\left\{S_{\Delta}\left(F_{E, f}\right)>\frac{t \lambda_{0}}{\sqrt{q}}\right\} .
$$

Soit $t>0$ tel que $\mu\left\{S_{\Delta}(f)>t\right\}>0$. Sans restreindre la généralité, on peut supposer $\left\|S_{u}(f)-S_{v}(f)\right\|_{2} \neq 0$ pour tout $u \neq v, u, v \in \Delta$. Posons alors

$$
\begin{aligned}
& \mathcal{D}=\left\{\frac{\left(S_{u}(f)-S_{v}(f)\right)^{2}}{\left\|S_{u}(f)-S_{v}(f)\right\|_{2, \mu}^{2}}: u \neq v, u, v \in \Delta \cup\{0\}\right\}, \\
& B=\left\{\forall z \in \mathcal{D}, \frac{1}{q} \sum_{T \in E} T z \leq 4\right\} .
\end{aligned}
$$

Soit $q=\left[Q t^{2}\right]+1$, où $Q$ ne dépend que de $\Delta$ et $f$, et sera déterminé en fin de démonstration. Posons aussi pour tout $z \in \mathcal{D}$,

$$
z_{q}=z \mathbf{1}_{\{z \leq q\}}, \quad z^{q}=z-z_{q}, \quad \bar{z}_{q}=z_{q}-\left\langle z_{q}, 1\right\rangle .
$$

Soit $0<h \leq 1$. Munis de ces données, considérons l'ensemble 


$$
\mathcal{F}=\left\{\bar{z}_{q}: z \in \mathcal{D}\right\} \cup\{\xi\}, \quad \text { où } \quad \xi=\frac{\mathbf{1}_{\left\{S_{\Delta}(f)>t\right\}}}{\mu\left\{S_{\Delta}(f)>t\right\}}-1 .
$$

Soit aussi

$$
0<\varepsilon \leq \min \left(1, \min \left\{\left\|z_{q}\right\|^{2} / q: z \in \mathcal{D}\right\}\right) .
$$

Nous appliquons tout d'abord la proposition 2.6 à l'ensemble $\mathcal{F}$ en choisissant comme suite $\left(\varepsilon_{p}\right)_{p}$ la suite constante égale à $\varepsilon$. Il en résulte que l'on peut déterminer un (une infinité en fait) ensemble $E$ séparant les éléments de $\mathcal{F}$. Plus exactement, les fonctions $\overline{z_{q} \circ T}, z \in \mathcal{D}, T \in E$, sont $\varepsilon$-orthogonales; entre autre,

$$
\forall z \in \mathcal{D}, T, U \in E, T \neq U, \quad\left|\left\langle\bar{z}_{q} \circ T, \bar{z}_{q} \circ U\right\rangle\right| \leq \varepsilon,
$$

et

$$
\forall T, U \in E, T \neq U, \quad|\langle\xi \circ T, \xi \circ U\rangle| \leq \varepsilon .
$$

Nous allons dans un premier temps évaluer la quantité

$$
\mu\left\{\frac{1}{q} \sum_{T \in E} T z>4\right\} \quad(z \in \mathcal{D}) .
$$

Tout d'abord, puisque pour tout $z \in \mathcal{D},\left|\left\langle z_{q}, 1\right\rangle\right| \leq\left\|z_{q}\right\|_{1} \leq\|z\|_{1}=1$, il s'ensuit que

$$
\begin{aligned}
\mu\left\{\frac{1}{q} \sum_{T \in E} T z>4\right\} & \leq \mu\left\{\frac{1}{q} \sum_{T \in E} T z_{q}>4-h\right\}+\mu\left\{\frac{1}{q} \sum_{T \in E} T z^{q}>h\right\} \\
& \leq \mu\left\{\frac{1}{q} \sum_{T \in E} T \bar{z}_{q}>3-h\right\}+\mu\left\{\frac{1}{q} \sum_{T \in E} T z^{q}>h\right\} \\
& =(\mathrm{I})+(\mathrm{II}) .
\end{aligned}
$$

Examinons (II) : soit $x \in X$ tel que

$$
\frac{1}{q} \sum_{T \in E} T z^{q}=\frac{1}{q} \sum_{T \in E} z(T x) \mathbf{1}_{\{z(T x)>q\}}>h .
$$

Alors, il existe $T \in E$ tel que $z(T x) \mathbf{1}_{\{z(T x)>q\}}>h$; et donc $z(T x)>q$. Autrement dit, $x \in \bigcup_{T \in E} T^{-1}\{z>q\}$. D'où

$$
\text { (II) } \leq q \mu\{z>q\} \text {. }
$$

Examinons (I) : comme on a choisi $E$ de sorte que les fonctions $T \bar{z}_{q}, T \in E$, $z \in \mathcal{D}$, soient $\varepsilon$-orthogonales, on a

$$
\left\|\frac{1}{q} \sum_{T \in E} T \bar{z}_{q}\right\|^{2} \leq \frac{\left\|\bar{z}_{q}\right\|^{2}}{q}+\varepsilon \leq \frac{\left\|z_{q}\right\|^{2}}{q}+\varepsilon .
$$

Puisque $\varepsilon \leq\left\|z_{q}\right\|^{2} / q$, il en résulte par une application directe de l'inégalité de Tchebycheff que 


$$
\text { (I) } \leq \frac{1}{(3-h)^{2}}\left(\frac{\left\|z_{q}\right\|^{2}}{q}+\varepsilon\right) \leq \frac{1}{2} \frac{\left\|z_{q}\right\|^{2}}{q} .
$$

En combinant les deux estimations précédentes, on obtient donc

$$
\mu\left\{\frac{1}{q} \sum_{T \in E} T z>4\right\} \leq \frac{\left\|z_{q}\right\|^{2}}{q}+q \mu\{z>q\} .
$$

Introduisons la fonctionnelle suivante de $z$ :

$$
\forall b>0, \quad D(z, b):=\frac{\int z^{2} \mathbf{1}_{\{z \leq b\}} d \mu}{b^{2}}+\mu\{z>b\} .
$$

Alors $D(z, \cdot)$ est une fonction décroissante, et comme $z$ est intégrable, $\lim _{b \rightarrow \infty} b D(z, b)=0$. Posons aussi

$$
\mathcal{D}(b)=\sup _{z \in \mathcal{D}} D(z, b)
$$

Comme $\Delta$ est fini, $\mathcal{D}(\cdot)$ possède les mêmes propriétés que $D(z, \cdot)$. On a donc établi que

$$
\mu\left\{\frac{1}{q} \sum_{T \in E} T z>4\right\} \leq q D(z, q) .
$$

Notons que cette dernière inégalité est vérifiée pour tout $z \in \mathcal{D}$. D'où

$$
\mu\left(B^{\mathrm{c}}\right) \leq \sharp(\Delta)^{2} q \mathcal{D}(q),
$$

puisque $\sharp(\mathcal{D})=\left(\begin{array}{c}\sharp(\Delta)+1 \\ 2\end{array}\right)$. Ceci montre donc que $\mu\left(B^{\mathrm{c}}\right)$ tend vers 0 quand $q$ tend vers l'infini. Par ailleurs, compte tenu du choix de $E$, les indicatrices

$$
\xi_{T}=\frac{\mathbf{1}_{\left\{S_{\Delta}(f) \circ T>t\right\}}}{\mu\left\{S_{\Delta}(f)>t\right\}}-1 \quad(T \in E),
$$

sont $\varepsilon$-orthogonales. Il en résulte que

$$
\begin{aligned}
\mu\left(\left\{S_{\Delta}(f) \circ T>t\right\} \cap\left\{S_{\Delta}(f) \circ V>t\right\}\right) \\
\quad \leq(1+\varepsilon) \mu\left\{S_{\Delta}(f)>t\right\}^{2} \leq 2 \mu\left\{S_{\Delta}(f)>t\right\}^{2} .
\end{aligned}
$$

A l'aide de l'inégalité de Paley-Zygmund : pour tout $g \in L^{2}(\mu)$ telle que $\mu\{g \geq 0\}=1$ et tout réel $\lambda \in[0,1]$,

$$
\mu\left\{g \geq \lambda \int g d \mu\right\} \geq(1-\lambda)^{2} \frac{\left(\int g d \mu\right)^{2}}{\int g^{2} d \mu}
$$

on en déduit donc

$$
\begin{aligned}
\mu\left\{\sup _{T \in E} T S_{\Delta}(f)>t\right\} & \geq \frac{\left(\mathbb{E} \sum \mathbf{1}_{\left\{T S_{\Delta}(f)>t\right\}}\right)^{2}}{\mathbb{E}\left(\sum \mathbf{1}_{\left\{T S_{\Delta}(f)>t\right\}}\right)^{2}} \geq \frac{q \mu\left\{S_{\Delta}(f)>t\right\}}{1+2 q \mu\left\{S_{\Delta}(f)>t\right\}} \\
& \geq \frac{1}{3} \min \left(1, q \mu\left\{S_{\Delta}(f)>t\right\}\right) .
\end{aligned}
$$

Nous allons majorer la quantité $\mu \times \mathbb{P}\left\{S_{\Delta}\left(F_{E, f}\right)>t \lambda_{0} / \sqrt{q}\right\}$ en procédant comme suit : 


$$
\mu \times \mathbb{P}\left\{S_{\Delta}\left(F_{E, f}\right)>\frac{t \lambda_{0}}{\sqrt{q}}\right\} \leq \mu\left(B^{\mathrm{c}}\right)+\int_{B} \mathbb{P}\left\{S_{\Delta}\left(F_{E, f}\right)>\frac{t \lambda_{0}}{\sqrt{q}}\right\} d \mu .
$$

Compte tenu de la propriété de commutation, $S_{\Delta}\left(F_{E, f}\right)=\sup _{n \in \Delta}\left|F_{E, S_{n}(f)}\right|$. Nous sommes donc ramenés à l'évaluation d'un maximum de processus gaussien presque sûrement borné, dont la fonction de covariance est comparable à celle du processus gaussien canonique $Z$, par construction même de $B$. L'inégalité de Tchebycheff, ainsi que les propriétés de comparaison des moments des semi-normes gaussiennes (voir par exemple [LT], Corollaire 3.2, p. 59) montrent que

$\mathbb{P}\left\{S_{\Delta}\left(F_{E, f}\right)>\frac{t \lambda_{0}}{\sqrt{q}}\right\} \leq \frac{q}{\lambda_{0}^{2} t^{2}} \mathbb{E} \sup _{n \in \Delta}\left|F_{E, S_{n}(f)}\right|^{2} \leq C_{1} \frac{q}{\lambda_{0}^{2} t^{2}}\left(\mathbb{E} \sup _{n \in \Delta}\left|F_{E, S_{n}(f)}\right|\right)^{2}$,

avec $C_{1}=\pi / 2$. Mais, compte tenu cette fois des propriétés de symétrie des lois gaussiennes, pour tout $m_{0} \in \Delta$,

$$
\begin{aligned}
\mathbb{E} \sup _{n \in \Delta}\left|F_{E, S_{n}(f)}\right| & \leq \mathbb{E} \sup _{n \in \Delta}\left|F_{E, S_{n}(f)}-F_{E, S_{m_{0}}(f)}\right|+\mathbb{E}\left|F_{E, S_{m_{0}}(f)}\right| \\
& \leq \mathbb{E} \sup _{n, m \in \Delta}\left|F_{E, S_{n}(f)}-F_{E, S_{m}(f)}\right|+\sqrt{2 / \pi}\left\|F_{E, S_{m_{0}}(f)}\right\|_{2, \mathbb{P}} \\
& =2 \mathbb{E} \sup _{n \in \Delta} F_{E, S_{n}(f)}+\sqrt{2 / \pi}\left\|F_{E, S_{m_{0}}(f)}\right\|_{2, \mathbb{P} .}
\end{aligned}
$$

L'intégration de cette quantité portant sur $B$, nous savons que pour tous $n, m \in \Delta$,

$$
\begin{aligned}
\left\|F_{E, S_{n}(f)}-F_{E, S_{m}(f)}\right\|_{2, \mathbb{P}}^{2} & =\frac{1}{q} \sum_{T \in E}\left(S_{n}(f \circ T)-S_{m}(f \circ T)\right)^{2} \\
& \leq 4\left\|S_{n}(f)-S_{m}(f)\right\|_{2, \mu}^{2}, \\
\left\|F_{E, S_{n}(f)}\right\|_{2, \mathbb{P}}^{2} & =\frac{1}{q} \sum_{T \in E}\left(S_{n}(f \circ T)\right)^{2} \leq 4\left\|S_{n}(f)\right\|_{2, \mu}^{2} .
\end{aligned}
$$

Par conséquent, en vertu du lemme de Slepian,

$$
\mathbb{E} \sup _{n \in \Delta} F_{E, S_{n}(f)} \leq 2 \mathbb{E} \sup _{n \in \Delta} Z\left(S_{n}(f)\right) \leq 2 \zeta(\Delta, f) .
$$

Et, en outre $\left\|F_{E, S_{m}(f)}\right\|_{2, \mathbb{P}} \leq 2\left\|S_{n}(f)\right\|_{2, \mu} \leq \sqrt{2 \pi} \zeta(\Delta, f)$. Ces différentes estimations montrent donc que sur $B$,

$$
\mathbb{E} \sup _{n \in \Delta}\left|F_{E, S_{n}(f)}\right| \leq \zeta(\Delta, f)(4+\sqrt{2 \pi} \sqrt{2 / \pi}) \leq 8 \zeta(\Delta, f) .
$$

Ainsi donc,

$$
\int_{B} \mathbb{P}\left\{S_{\Delta}\left(F_{E, f}\right)>\frac{t \lambda_{0}}{\sqrt{q}}\right\} d \mu \leq 32 \pi \frac{q \zeta(\Delta, f)^{2}}{\lambda_{0}^{2} t^{2}} \leq 101 \frac{q \zeta(\Delta, f)^{2}}{\lambda_{0}^{2} t^{2}} .
$$


D'où

$$
\frac{1}{3} \min \left(1, q \mu\left\{S_{\Delta}(f)>t\right\}\right) \leq 4 \sharp(\Delta)^{2} q \mathcal{D}(q)+404 \frac{q \zeta(\Delta, f)^{2}}{\lambda_{0}^{2} t^{2}} .
$$

On suppose $t^{2} \geq Q^{-1}$. Comme $q=\left[Q t^{2}\right]+1$, on a donc $Q t^{2} \leq q \leq 2 Q t^{2}$. Il en résulte que

$$
\begin{aligned}
\min \left(1, Q t^{2} \mu\left\{S_{\Delta}(f)>t\right\}\right) & \leq \min \left(1, q \mu\left\{S_{\Delta}(f)>t\right\}\right) \\
& \leq 24 \sharp(\Delta)^{2} Q t^{2} \mathcal{D}\left(Q t^{2}\right)+\frac{2424}{\lambda_{0}^{2}} Q \zeta(\Delta, f)^{2} .
\end{aligned}
$$

Choisissons $Q=\lambda_{0}^{2} /\left(4848 \zeta(\Delta, f)^{2}\right)$, puis $t$ suffisamment grand pour que

$$
24 \sharp(\Delta)^{2} Q t^{2} \mathcal{D}\left(Q t^{2}\right) \leq 1 / 2 .
$$

Alors $\min \left(1, Q t^{2} \mu\left\{S_{\Delta}(f)>t\right\}\right) \leq 1$. Donc

$$
\mu\left\{S_{\Delta}(f)>t\right\} \leq \frac{1}{Q t^{2}}=\frac{4848}{\lambda_{0}^{2}} \cdot \frac{\zeta(\Delta, f)^{2}}{t^{2}} .
$$

La constante $K$ de l'énoncé est donc égale à $4848 / \lambda_{0}^{2}$. D'où le théorème 1.8 .

6. Appendice. Soit $\mathcal{E}=\left\{T_{j}: j \geq 1\right\}$ une famille de transformations mesurables de $X$, préservant $\mu$, et faiblement mélangeante :

$$
\forall f, g \in L^{2}(\mu), \quad \lim _{n \rightarrow \infty} \frac{1}{n} \sum_{k=1}^{n}\left|\left\langle T_{k} f, g\right\rangle-\mu(f) \mu(g)\right|=0 .
$$

La partie de la démonstration du théorème 1.8 concernant l'évaluation de l'ensemble $B$ utilise le fait que si $z \in L^{2}(\mu) \cap \mathbf{1}_{\mu}^{\perp}$, et $N \geq 1$ est un entier arbitraire, alors on peut trouver des entiers $u_{1}, \ldots, u_{N}$ tels que si $S(z)=$ $z \circ T_{u_{1}}+\ldots+z \circ T_{u_{N}}$, l'on ait

$$
\forall z \in \Delta, \quad\|S(z)\|_{2, \mu}^{2} \leq 2 N\|z\|_{2, \mu}^{2} .
$$

Il est naturel de chercher à savoir si cette propriété s'étend à tout espace $L^{p}(\mu), 2<p<\infty$. La proposition ci-dessous montre que tel est le cas, et donne l'existence d'un analogue des inégalités de H. P. Rosenthal ([LT], inégalité 10.6 , p. 285) dans le cadre du mélange faible.

Proposition 6.1. Soient un entier $p \geq 2$ et un réel $\varepsilon>0$. Soient $\Delta \subset L^{p}(\mu)$ un ensemble fini, ainsi qu'un entier $N \geq 1$. On peut trouver des entiers $u_{1}, \ldots, u_{N}$ tels que si $S(z)=z \circ T_{u_{1}}+\ldots+z \circ T_{u_{N}}$, l'on ait

$$
\forall z \in \Delta, \quad \int_{X} S(z)^{p} d \mu \leq(1+\varepsilon)^{p}(2 p N)^{p / 2} \int_{X} z^{p} d \mu .
$$

La démonstration va résulter de la proposition ci-dessous : 
Proposition 6.2. Soient $f, g \in L^{\infty}(\mu)$. Soient $X$ et $Y$ deux variables aléatoires indépendantes, telles que $X \sim f$ et $Y \sim g$. Soit aussi $F: \mathbb{R}^{2} \rightarrow \mathbb{R}$ continue. Alors pour tout $\varepsilon>0$, on peut choisir une suite $\mathbf{S}$ d'entiers positifs de densité 1 , telle que pour tout $u \in \mathbf{S}$,

$$
\left|\int_{X} F\left(f, T_{u} g\right) d \mu-\mathbb{E} F(X, Y)\right| \leq \varepsilon .
$$

Montrons comment en déduire la proposition 6.1. Soient $z \in L^{\infty}(\mu)$ et $\alpha>0$. Posons $Z=\int(1+|z|)^{p} d \mu$. Soient $X_{1}, X_{2}, \ldots$ une suite de variables aléatoires indépendantes, de même loi que $z$. Soit aussi $F(x, y)=(x+y)^{l}$, $l \leq p$. En appliquant $p$ fois la proposition précédente avec les choix $f=g$ $=z$, on établit l'existence d'une suite d'entiers $\mathbf{S}_{1}$ de densité 1 , telle que pour tout $u \in \mathbf{S}_{1}$,

$$
\forall l \leq p, \quad\left|\int\left(z+T_{u} z\right)^{l} d \mu-\mathbb{E}\left(X_{1}+X_{2}\right)^{l}\right| \leq \alpha .
$$

$\mathrm{Au}$ cran suivant, on applique la proposition 6.2 avec les choix $f=z+T_{u} z$, $g=z$ et $X, Y$ indépendantes avec $X \sim f$ et $Y \sim g$. Pour tous $u \in \mathbf{S}_{1}$ et $v$ appartenant à une suite $\mathbf{S}_{2}$ (dépendant de $u$ ) de densité 1 , on a

$$
\forall l \leq p, \quad\left|\int\left(z+T_{u} z+T_{v} z\right)^{l} d \mu-\mathbb{E}(X+Y)^{l}\right| \leq \alpha .
$$

Mais

$$
\mathbb{E}(X+Y)^{l}=\sum_{k=0}^{l} C_{l}^{k} \mathbb{E} X^{k} \mathbb{E} Y^{l-k}=\sum_{k=0}^{l} C_{l}^{k} \int\left(z+T_{u} z\right)^{k} d \mu \int z^{l-k} d \mu .
$$

Donc

$$
\begin{aligned}
\mid \mathbb{E}(X+Y)^{l}- & \mathbb{E}\left(X_{1}+X_{2}+X_{3}\right)^{l} \mid \\
& =\left|\mathbb{E}(X+Y)^{l}-\sum_{k=0}^{l} C_{l}^{k} \mathbb{E}\left(X_{1}+X_{2}\right)^{k} \int z^{l-k} d \mu\right| \\
& =\left|\sum_{k=0}^{l} C_{l}^{k}\left(\int\left(z+T_{u} z\right)^{k} d \mu-\mathbb{E}\left(X_{1}+X_{2}\right)^{k}\right) \int z^{l-k} d \mu\right| \\
& \leq \sum_{k=0}^{l} C_{l}^{k}\left|\int\left(z+T_{u} z\right)^{k} d \mu-\mathbb{E}\left(X_{1}+X_{2}\right)^{k}\right| \cdot\left|\int z^{l-k} d \mu\right| \\
& \leq \alpha \sum_{k=0}^{l} C_{l}^{k} \int\left|z^{l-k}\right| d \mu=\alpha \int(1+|z|)^{l} d \mu \leq \alpha Z .
\end{aligned}
$$

On en déduit donc pour tous $u \in \mathbf{S}_{1}, v \in \mathbf{S}_{2}$ et $l \leq p$,

$$
\left|\int\left(z+T_{u} z+T_{v} z\right)^{l} d \mu-\mathbb{E}\left(X_{1}+X_{2}+X_{3}\right)^{l}\right| \leq \alpha(1+Z) .
$$


Il suffit alors d'itérer l'argument précédent. Pour tout entier $N \geq 1$, on montre ainsi qu'il existe $N$ suites $\mathbf{S}_{1}, \ldots, \mathbf{S}_{N}$ de densité 1 telles que pour tous $u_{i} \in \mathbf{S}_{i}, i=1, \ldots, N$ et $l \leq p$,

$$
\left|\int\left(\sum_{i=1}^{N} T_{u_{i}} z\right)^{l} d \mu-\mathbb{E}\left(\sum_{i=1}^{N} X_{i}\right)^{l}\right| \leq \alpha\left[\sum_{\lambda=0}^{(N-2)^{+}} Z^{\lambda}\right] .
$$

Notons que pour tout $i=1,2, \ldots$, la suite $\mathbf{S}_{i}$ dépend de $u_{1}, \ldots, u_{i-1}$. En choisissant convenablement $\alpha$ en fonction de $\varepsilon, N, z$ et $p$, on aura donc entre autres

$$
\int\left(\sum_{i=1}^{N} T_{u_{i}} z\right)^{p} d \mu \leq \mathbb{E}\left(\sum_{i=1}^{N} X_{i}\right)^{p}+\varepsilon .
$$

On en déduit par approximation le même résultat pour $z \in L^{p}(\mu)$, puis pour toute partie finie $\Delta$ de $L^{p}(\mu)$. La proposition 6.1 découle alors de ce résultat et de l'estimation par H. P. Rosenthal de $\mathbb{E}\left(\sum_{i=1}^{N} X_{i}\right)^{p}$. Nous renvoyons pour ce dernier point à [LT], inégalité 10.6, p. 285. Il nous reste donc à établir la proposition 6.2.

Démonstration de la proposition 6.2. Il suffit de l'établir pour $F(x, y)=$ $\sum_{k, l=1}^{M} a_{k, l} x^{k} y^{l}$. On en déduira alors le cas général à l'aide du théorème de Stone-Weierstrass. Soient $\varepsilon>0$ et $f, g \in L^{\infty}(\mu)$. Nous avons donc à considérer l'expression suivante :

$$
\int F\left(f, T_{u} g\right) d \mu=\sum_{k, l=1}^{M} a_{k, l} \int f^{k} T_{u} g^{l} d \mu .
$$

Or $\mathcal{E}$ est faiblement mélangeante. En vertu du théorème de Koopmann, il existe une suite $\mathbf{S}$ de densité 1 telle que

$$
\lim _{\mathbf{S} \ni u \rightarrow \infty} \int f^{k} T_{u} g^{l} d \mu=\int f^{k} d \mu \cdot \int g^{l} d \mu .
$$

On en déduit par induction, pour tout $\varepsilon^{\prime}>0$, l'existence d'une suite $\mathbf{S}$ de densité 1 telle que

$$
\forall u \in \mathbf{S}, \forall k, l=1, \ldots, M, \quad\left|\int f^{k} T_{u} g^{l} d \mu-\int f^{k} d \mu \cdot \int g^{l} d \mu\right| \leq \varepsilon^{\prime} .
$$

Choisissons $\varepsilon^{\prime}=\varepsilon /\left(\sum_{k, l=1}^{M}\left|a_{k, l}\right|\right)$. Alors, l'estimation précédente fournit

$$
\begin{aligned}
\left|\int F\left(f, T_{u} g\right) d \mu-\mathbb{E} F(X, Y)\right| & =\left|\int F\left(f, T_{u} g\right) d \mu-\sum_{k, l=1}^{M} a_{k, l} \int f^{k} d \mu \cdot \int g^{l} d \mu\right| \\
& \leq \sum_{k, l=1}^{M}\left|a_{k, l}\right|\left|\int f^{k} T_{u} g^{l} d \mu-\int f^{k} d \mu \cdot \int g^{l} d \mu\right| \leq \varepsilon
\end{aligned}
$$


Le cas général s'ensuit immédiatement. En effet, en vertu du théorème de Stone-Weierstrass, on peut trouver un polynôme $P(x, y)$ tel que $\|F-P\|_{\infty}$ $\leq \varepsilon$. A l'aide de l'inégalité du triangle, et en appliquant l'étape précédente à $P$, on en déduit l'existence d'une suite $\mathbf{S}$ de densité 1 telle que pour tout $u \in \mathbf{S}$,

$$
\left|\int F\left(f, T_{u} g\right) d \mu-\mathbb{E} F(X, Y)\right| \leq 2 \varepsilon+\left|\int P\left(f, T_{u} g\right) d \mu-\mathbb{E} P(X, Y)\right| \leq 3 \varepsilon
$$

Remerciements. L'auteur remercie vivement le referee pour une lecture attentive de la version initiale de ce travail et pour ses suggestions permettant de préciser l'ordre de grandeur des constantes universelles, ainsi que pour avoir proposé une autre démonstration de la proposition 6.1, que nous avons suivi ici.

\section{Références}

[BJ] A. Bellow and R. Jones, A Banach principle for $L^{\infty}$, Adv. Math. 120 (1996), 155-172.

[Bo1] J. Bourgain, Almost sure convergence and bounded entropy, Israel J. Math. 63 (1988), 79-95.

[K] U. Krengel, Ergodic Theorems, de Gruyter, 1989.

[LT] M. Ledoux and M. Talagrand, Probability in Banach Spaces, Springer, 1990.

[P] H. O. Pollak, A remark on "elementary inequalities for Mill's ratio" by Yûsaku Komatu, Rep. Statist. Appl. Res. Un. Jap. Sci. Engrs. 4 (1956), 110.

[RW] J. J. Ruch et M. Weber, Quelques résultats dans la théorie des sommes de Riemann, Exposition. Math. 15 (1997), 279-288.

[W1] M. Weber, GC sets, Stein's elements and matrix summation methods, Prépublication IRMA no 027, Université de Strasbourg, 1993.

[W2] -,$G B$ and $G C$ sets in ergodic theory, dans : Probability in Banach Spaces, 9 (Sandjberg, 1993), Progr. Probab. 35, Birkhäuser, Basel, 1994, 129-151.

[W3] - The Stein randomization procedure, Rend. Mat. 16 (1996), 569-605.

[W4] - Entropie métrique et convergence presque partout, Travaux en Cours 58, Hermann, Paris, 1998.

Mathématique (IRMA)

Université Louis-Pasteur

7, rue René Descartes

67084 Strasbourg Cedex, France

E-mail: weber@math.u-strasbg.fr

Received 26 January 1999;

in revised form 2 June 2000 